\title{
Periodic problems with double resonance
}

\author{
Giuseppina Barletta and Nikolaos S. Papageorgiou
}

\begin{abstract}
We consider a second order periodic problem with resonance both at infinity and at zero. Combining variational methods together with Morse theory, we produce six nontrivial solutions for the periodic problem.
\end{abstract}

Mathematics Subject Classification (2000). 34C25, 58E05.

\section{Introduction}

In this paper, we deal with the following second order periodic problem:

$$
\left\{\begin{array}{l}
-u^{\prime \prime}(t)=f(t, u(t)) \text { a.e. on } T=[0, b] \\
u(0)=u(b), u^{\prime}(0)=u^{\prime}(b)
\end{array}\right.
$$

In this problem the reaction term $f(t, x)$ is jointly measurable and $C^{1}$ in the $x$-variable. The aim of this work is to prove a multiplicity theorem when the problem is resonant both at infinity and at zero. Such problems are known in the literature as "doubly resonant". Resonant Dirichlet equations, but with the resonance only at infinity and only with respect to the first two eigenvalues, were studied by Dancer and Gupta [7], Gupta [10], Iannacci and Nkashama [11] and Sanchez [18]. Doubly resonant Dirichlet equations of higher parts of the spectrum were investigated by $\mathrm{Su}$ and $\mathrm{Li}$ [19] and $\mathrm{Zou}$ [22]. For periodic equations, we have the recent work of $\mathrm{Su}$ and Zhao [20]. With the exception of $\mathrm{Su}$ and $\mathrm{Li}$ [19], all the other works produce two nontrivial solutions. Su and $\mathrm{Li}$ [19] dealing with Dirichlet equations, produce six nontrivial solutions. Here under more general hypotheses on the reaction $f(t, x)$, we produce six nontrivial solutions for the periodic problem (1). Our approach combines variational techniques based on the critical point theory together with Morse theory. In the next section, for the convenience of the reader, we recall the main mathematical tools that we will use in this paper. 


\section{Mathematical background-hypotheses}

Let $X$ be a Banach space and $X^{*}$ its topological dual. By $\langle\cdot, \cdot\rangle$ we denote the duality brackets for the pair $\left(X^{*}, X\right)$. Let $\varphi: X \rightarrow \mathbb{R}$ be a $C^{1}$ function. We say that $\varphi$ satisfies the "Cerami condition" (the "C-condition" for short), if every sequence $\left\{x_{n}\right\}_{n \geq 1} \subseteq X$ such that

$\left\{\varphi\left(x_{n}\right)\right\} \subseteq \mathbb{R}$ is bounded and $\left(1+\left\|x_{n}\right\|\right) \varphi^{\prime}\left(x_{n}\right) \rightarrow 0$ in $X^{*}$ as $n \rightarrow \infty$,

has a strongly convergent subsequence. Using this compactness type condition, we have the following minimax theorem for the critical values of a $C^{1}$-functional, known in the literature as the "mountain pass theorem".

Theorem 2.1. If $X$ is a Banach space, $\varphi: X \rightarrow \mathbb{R}$ is $C^{1}$, satisfies the $C$-condition, $x_{0}, x_{1} \in X, 0<\rho<\left\|x_{1}-x_{0}\right\|, \max \left\{\varphi\left(x_{0}\right), \varphi\left(x_{1}\right)\right\}<\inf [\varphi(x)$ : $\left.\left\|x-x_{0}\right\|=\rho\right]=\eta_{\rho}$, and

$c=\inf _{\gamma \in \Gamma} \max _{t \in[0,1]} \varphi(\gamma(t))$ where $\Gamma:=\left\{\gamma \in C^{0}([0,1], X): \gamma(0)=x_{0}, \gamma(1)=x_{1}\right\}$,

then $c \geq \eta_{\rho}$ and $c$ is a critical value of $\varphi$.

For $\varphi \in C^{1}(X)$ and $c \in \mathbb{R}$, we introduce the following notation:

$$
\begin{aligned}
\varphi^{c} & =\{x \in X: \varphi(x) \leq c\}, \quad K^{\varphi}=\left\{x \in X: \varphi^{\prime}(x)=0\right\} \quad \text { and } \\
K_{c}^{\varphi} & =\left\{x \in K^{\varphi}: \varphi(x)=c\right\} .
\end{aligned}
$$

If $\left(Y_{1}, Y_{2}\right)$ is a topological pair with $Y_{2} \subseteq Y_{1} \subseteq X$, then for every integer $k \geq 0$, by $H_{k}\left(Y_{1}, Y_{2}\right)$ we denote the $k$ th-relative singular homology group for the pair $\left(Y_{1}, Y_{2}\right)$ with integer coefficients. The critical groups of $\varphi$ at an isolated critical point $x_{0} \in X$ with $\varphi\left(x_{0}\right)=c$ (i.e., $\left.x_{0} \in K_{c}^{\varphi}\right)$, are defined by

$$
C_{k}\left(\varphi, x_{0}\right)=H_{k}\left(\varphi^{c} \cap \mathcal{U}, \varphi^{c} \cap \mathcal{U} \backslash\left\{x_{0}\right\}\right) \text {, for all } k \geq 0,
$$

where $\mathcal{U}$ is a neighborhood of $x_{0}$ such that $K^{\varphi} \bigcap \varphi^{c} \bigcap \mathcal{U}=\left\{x_{0}\right\}$ (see $[6,13]$ ). The excision property of singular homology, implies that the above definition is independent of the particular choice of neighborhood $\mathcal{U}$ of $x_{0}$. Assume that $\varphi \in C^{1}(X)$ satisfies the C-condition and $-\infty<\inf \varphi\left(K^{\varphi}\right)$. Let $c<\inf \varphi\left(K^{\varphi}\right)$. The critical groups of $\varphi$ at infinity are defined by

$$
C_{k}(\varphi, \infty)=H_{k}\left(X, \varphi^{c}\right) \text { for all } k \geq 0,(\text { see }[5]) .
$$

The second deformation theorem (see, for example [15, p. 274]), implies that the above definition is independent of the particular choice of the level $c<$ $\inf \varphi\left(K^{\varphi}\right)$.

If $K^{\varphi}=\left\{x_{0}\right\}$, then we have $C_{k}(\varphi, \infty)=C_{k}\left(\varphi, x_{0}\right)$ for all $k \geq 0$. The next result is useful in computing the critical groups at infinity. It is a slight generalization of a result of Perera and Schechter [17], suitable for functions $\varphi \in C^{1}(X)$ which satisfy the C-condition (see [12]).

Proposition 2.1. If $H$ is a Hilbert space, $\left\{\varphi_{t}\right\}_{t \in[0,1]} \subseteq C^{1}(X), \varphi_{t}^{\prime}$ and $\partial_{t} \varphi_{t}$ both are locally Lipschitz, $\varphi_{0}$ and $\varphi_{1}$ satisfy the $C$-condition, and there exist $a \in \mathbb{R}$ and $\delta>0$ such that

$$
\varphi_{t}(u) \leq a \Rightarrow(1+\|u\|)\left\|\varphi_{t}^{\prime}(u)\right\| \geq \delta \quad \text { for all } t \in[0,1]
$$

then $C_{k}\left(\varphi_{0}, \infty\right)=C_{k}\left(\varphi_{1}, \infty\right)$ for all $k \geq 0$. 
Remark 2.1. Note that in particular, if there exists $R>0$ such that

$$
\begin{aligned}
& \inf \left[(1+\|u\|)\left\|\varphi_{t}^{\prime}(u)\right\|: t \in[0,1],\|u\|>R\right]>0, \\
& \inf \left[\left\|\varphi_{t}(u)\right\|: t \in[0,1],\|u\| \leq R\right]>-\infty,
\end{aligned}
$$

then $C_{k}\left(\varphi_{0}, \infty\right)=C_{k}\left(\varphi_{1}, \infty\right)$ for all $k \geq 0$.

We set

$$
m_{k}(x)=\operatorname{rank} C_{k}(\varphi, x) \text { for } x \in K^{\varphi} \text { and } \beta_{k}=\operatorname{rank} C_{k}(\varphi, \infty) \text { for all } k \geq 0
$$

and we introduce the polynomials

$$
P(t, x)=\sum_{k \geq 0} m_{k}(x) t^{k} \quad \text { and } \quad P(t, \infty)=\sum_{k \geq 0} \beta_{k} t^{k} \quad \text { for all } t \in \mathbb{R} .
$$

The Morse relation says that there exists a polynomial $Q(t)$ with nonnegative integer coefficients such that

$$
\sum_{x \in K^{\varphi}} P(t, x)=P(t, \infty)+(1+t) Q(t) \quad \text { for all } t \in \mathbb{R} .
$$

Also, if $\widehat{m}_{k}=\sum_{x \in K^{\varphi}} m_{k}(x)$ (the Morse-type numbers for $\varphi$ ) and $\beta_{k}$ (the Betti-type numbers for $\varphi$ ) are as above, then we have the Morse inequality

$$
\sum_{k=0}^{q}(-1)^{q-k} \widehat{m}_{k} \geq \sum_{k=0}^{q}(-1)^{q-k} \beta_{k}, \quad q \in \mathbb{N} .
$$

Let $X=H$ be a Hilbert space and let $x_{0} \in H$ be an isolated critical point of $\varphi$. Let $\mathcal{U}$ be a neighborhood of $\varphi$ and assume that $\varphi \in C^{2}(\mathcal{U})$. The "Morse index of $x_{0}$ ", is the supremum of the dimension of the subspaces of $H$, on which $\varphi^{\prime \prime}\left(x_{0}\right)$ is negative definite. The "nullity of $x_{0}$ ", is the dimension of the kernel of $\varphi^{\prime \prime}\left(x_{0}\right)$. We say that $x_{0}$ is "nondegenerate", if the nullity of $x_{0}$ is zero (i.e., $\varphi^{\prime \prime}\left(x_{0}\right)$ is invertible). Suppose that $\varphi^{\prime \prime}\left(x_{0}\right)$ is a Fredholm operator. We have the following facts about the critical groups of $\varphi$ at $x_{0}$ :

(a) if $x_{0}$ is "nondegenerate" critical point of $\varphi$, then

$$
C_{k}\left(\varphi, x_{0}\right)=\delta_{k, \mu_{0}} \mathbb{Z} \text { for all } k \geq 0,
$$

where $\mu_{0}$ is the Morse index of $x_{0}$.

(b) if $x_{0}$ is possibly degenerate with Morse index $\mu_{0}$ and nullity $\nu_{0}$, then

$$
C_{k}\left(\varphi, x_{0}\right)=0 \text { for all } k \notin\left[\mu_{0}, \mu_{0}+\nu_{0}\right] \text {. }
$$

Part (b) is known as the "Gromoll-Meyer Theorem" (see [9]).

Next, consider the linear eigenvalue problem:

$$
-u^{\prime \prime}(t)=\lambda u(t) \text { on } T=[0, b], \quad u(0)=u(b), \quad u^{\prime}(0)=u^{\prime}(b) .
$$

It is well known (see, for example, [15, p. 309]), that the eigenvalues of (3) are $\left\{\lambda_{n}=\left(\frac{2 \pi n}{b}\right)^{2}\right\}_{n \geq 0}$. All eigenvalues $\lambda_{n}$ for $n \geq 1$ have multiplicity equal to 2 and the corresponding eigenfunctions are nodal (i.e., sign changing). Also, $\lambda_{0}=0$ has multiplicity 1 and the corresponding eigenspace is $\mathbb{R}$ (the space of constant functions). In what follows, by $E\left(\lambda_{k}\right)$ we denote the eigenspace corresponding to the eigenvalue $\lambda_{k}, k \geq 0$. 
In the analysis of problem (1), we will use the space

$$
W_{\text {per }}^{1,2}(0, b)=\left\{u \in W^{1,2}(0, b): u(0)=u(b)\right\} .
$$

It is well known that $W_{p e r}^{1,2}(0, b)$ is compactly embedded in $C(T)$. In what follows, by $\|\cdot\|$ we denote the norm of $W_{p e r}^{1,2}(0, b)$ and by $\langle\cdot, \cdot\rangle$ the duality brackets for the pair $\left(W_{\text {per }}^{1,2}(0, b)^{*}, W_{\text {per }}^{1,2}(0, b)\right)$. Also in the sequel for notational economy, we set $W=W_{p e r}^{1,2}(0, b)$. For every integer $k \geq 1$, we have the orthogonal direct sum decomposition

$$
W=\bar{W}_{k-1} \oplus E\left(\lambda_{k}\right) \oplus \widehat{W}_{k+1},
$$

where $\bar{W}_{k-1}=\oplus_{i=0}^{k-1} E\left(\lambda_{i}\right)$ and $\widehat{W}_{k+1}=\bar{\oplus}_{i \geq k+1} E\left(\lambda_{i}\right)$. So, every $u \in W$ admits the unique sum decomposition

$$
u=\bar{u}+u^{0}+\widehat{u}, \quad \text { with } \quad \bar{u} \in \bar{W}_{k-1}, \quad u^{0} \in E\left(\lambda_{k}\right), \quad \widehat{u} \in \widehat{W}_{k+1} .
$$

We will also use the space $\widehat{C}(T)=C^{1}(T) \bigcap W=\left\{u \in C^{1}(T): u(0)=u(b)\right\}$. Note that $\widehat{C}(T)$ is an ordered Banach space with positive cone

$$
C_{+}=\{u \in \widehat{C}(T): u(t) \geq 0 \text { for all } t \in T\} .
$$

This cone has a nonempty interior given by

$$
\text { int } C_{+}=\left\{u \in C_{+}: u(t)>0 \quad \text { for all } t \in T\right\} .
$$

Finally let $A: W \rightarrow W^{*}$ be defined by

$$
\langle A(u), y\rangle=\int_{0}^{b} u^{\prime}(t) y^{\prime}(t) d t \quad \text { for all } u, y \in W .
$$

Evidently $A \in \mathcal{L}\left(W, W^{*}\right)$.

Next let us introduce the hypotheses on the reaction term $f(t, x)$ :

$H: f: T \times \mathbb{R} \rightarrow \mathbb{R}$ is a function such that

(i) for all $x \in \mathbb{R}, t \rightarrow f(t, x)$ is measurable;

(ii) for a.a. $t \in T, x \rightarrow f(t, x)$ is $C^{1}$ and $f(t, 0)=0$;

(iii) for a.a. $t \in T$ and all $x \in \mathbb{R}$, we have

$$
\left|f_{x}^{\prime}(t, x)\right| \leq a(t)\left(1+|x|^{r-2}\right) \text { with } a \in L^{\infty}(T)_{+}, 2 \leq r<+\infty ;
$$

(iv) there exist an integer $m \geq 1, \alpha \in(0,1)$ and $\theta_{\infty} \in L^{\infty}(T), \theta_{\infty} \leq 0$ a.e. on $T, \theta_{\infty} \neq 0$ such that

$$
\lim _{|x| \rightarrow \infty} \frac{f(t, x)}{x}=\lambda_{m} \quad \text { uniformly for a.a. } t \in T
$$

and if $f_{\infty}(t, x)=f(t, x)-\lambda_{m} x$, then

$\lim _{|x| \rightarrow \infty} \frac{f_{\infty}(t, x)}{|x|^{\alpha}}=0$ and $\limsup _{|x| \rightarrow \infty} \frac{f_{\infty}(t, x) x}{|x|^{2 \alpha}} \leq \theta_{\infty}(t) \quad$ uniformly for a.a. $t \in T ;$

(v) there exist an integer $i \geq 1, i \neq m, \beta>1$ and $\theta_{0} \in L^{\infty}(T), \theta_{0} \leq 0$ a.e. on $T, \theta_{0} \neq 0$ such that

$$
\lim _{|x| \rightarrow 0} \frac{f(t, x)}{x}=\lambda_{i} \quad \text { uniformly for a.a. } t \in T
$$


and if $f_{0}(t, x)=f(t, x)-\lambda_{i} x$, then $\lim _{|x| \rightarrow 0} \frac{f_{0}(t, x)}{|x|^{\beta}}=0$ and $\limsup _{|x| \rightarrow 0} \frac{f_{0}(t, x) x}{|x|^{2 \beta}} \leq \theta_{0}(t)$ uniformly for a.a. $t \in T ;$

(vi) there exist numbers $\xi_{-}<0<\xi_{+}$such that $f\left(t, \xi_{+}\right) \leq 0 \leq f\left(t, \xi_{-}\right)$for a.a. $t \in T$.

Remark 2.2. Hypotheses $H(\mathrm{iv})$ and (v) imply that we have resonance at infinity and at zero, respectively. These hypotheses classify the problem as "double resonant".

Example 2.1. The following function $f(x)$ satisfies hypotheses $H$ (for the sake of simplicity, we drop the $t$-dependance)

$$
f(x)= \begin{cases}\lambda_{i} x-\eta_{0}|x|^{q-2} x & \text { if }|x| \leq 1 \\ \lambda_{m} x-\eta_{\infty}|x|^{\tau-2} x+\mu \cdot \operatorname{sgn} x & \text { if }|x|>1\end{cases}
$$

where $1<\tau<2<q, \eta_{0} \geq \lambda_{i}, \mu=\eta_{0}(q-2)+\eta_{\infty}(2-\tau)>0$. For this particular reaction function we can take $\alpha \in(\tau-1,1)$ (see $H($ iv) $), \beta \in\left(\frac{q}{2}, q-1\right)$ (see $H(\mathrm{v}))$ and $\xi_{-}=-1, \xi_{+}=1($ see $H(\mathrm{vi}))$.

Let $\varphi: W \rightarrow \mathbb{R}$ be the energy functional for problem (1) defined by

$$
\varphi(u)=\frac{1}{2}\left\|u^{\prime}\right\|_{2}^{2}-\int_{0}^{b} F(t, u(t)) d t \quad \text { for all } u \in W,
$$

where $F(t, x)=\int_{0}^{x} f(t, s) d s$. Hypotheses $H$ imply $\varphi \in C^{2}(W)$. We will also need the notion of upper and lower solutions for problem (1).

Definition 2.1. (a) A function $\bar{u} \in W^{1,2}(0, b)$ is an "upper solution" for problem (1), if

$$
\int_{0}^{b} \bar{u}^{\prime} \psi^{\prime} d t \geq \int_{0}^{b} f(z, \bar{u}) \psi d t \quad \text { for all } \psi \in C_{c}^{1}(0, b), \psi \geq 0
$$

and $\bar{u}(b) \geq \bar{u}(0)$.

(b) A function $\underline{u} \in W^{1,2}(0, b)$ is a "lower solution" for problem (1), if

$$
\int_{0}^{b} \underline{u}^{\prime} \psi^{\prime} d t z \leq \int_{0}^{b} f(z, \underline{u}) \psi d t \quad \text { for all } \psi \in C_{c}^{1}(0, b), \psi \geq 0
$$

and $\underline{u}(b) \leq \underline{u}(0)$.

\section{C-condition, critical groups}

In this section we show the C-condition for the energy functional $\varphi \in C^{2}(W)$ and we compute its critical groups at the origin and at infinity. To this end we prove some auxiliary results which can be deduced from hypotheses $H(\mathrm{iv}),(\mathrm{v})$.

So, let $\lambda_{i}>0$ be the eigenvalue postulated by hypothesis $H(\mathrm{v})$ and consider the orthogonal direct sum decomposition of the space $W$ corresponding to it, i.e.,

$$
W=\bar{W}_{i-1} \oplus E\left(\lambda_{i}\right) \oplus \widehat{W}_{i+1} .
$$


Then for every $u \in W$, we have $u=\bar{u}+u^{0}+\widehat{u}$, where $\bar{u} \in \bar{W}_{i-1}, u^{0} \in E\left(\lambda_{i}\right)$, $\widehat{u} \in \widehat{W}_{i+1}$.

Proposition 3.1. If $\left\{u_{n}\right\}_{n \geq 1} \subseteq W$ is a sequence such that $\left\|u_{n}\right\| \rightarrow 0$ and $\frac{\widehat{u}_{n}+\bar{u}_{n}}{\left\|u_{n}\right\|} \rightarrow 0$ in $W$, then $\lim \sup _{n \rightarrow \infty} \int_{0}^{b} \frac{f_{0}\left(t, u_{n}\right) u_{n}}{\left\|u_{n}\right\|^{2 \beta}} d t<0$.

Proof. In what follows, by $|\cdot|$ we denote the Lebesgue measure on $\mathbb{R}$. From Bartolo et al. [3], we know that given $\varepsilon>0$, we can find $\mu_{1}(\varepsilon) \in(0,1)$, $\mu_{2}(\varepsilon)>0$ large such that

$$
\begin{gathered}
\left|\left\{t \in T:\left|u^{0}(t)\right|<\mu_{1}\left\|u^{0}\right\|\right\}\right|<\varepsilon \text { for all } u^{0} \in E\left(\lambda_{i}\right) \backslash\{0\} \text { and } \\
\qquad\left\{t \in T:|\widehat{u}(t)+\bar{u}(t)|>\mu_{2}\|\widehat{u}+\bar{u}\|\right\} \mid \\
<\mu_{1}^{2 \beta} \varepsilon \text { for all } \widehat{u} \in \widehat{W}_{i+1}, \bar{u} \in \bar{W}_{i-1}, \widehat{u}+\bar{u} \neq 0 .
\end{gathered}
$$

For every $n \geq 1$, we introduce the following sets

$$
\begin{aligned}
& T_{1 n}=\left\{t \in T:\left|u_{n}^{0}(t)\right| \geq \mu_{1}\left\|u_{n}^{0}\right\|\right\} \text { and } \\
& T_{2 n}=\left\{t \in T:\left|\widehat{u}_{n}(t)+\bar{u}_{n}(t)\right| \leq \mu_{2}\left\|\widehat{u}_{n}+\bar{u}_{n}\right\|\right\} .
\end{aligned}
$$

Evidently we have

$$
\begin{array}{r}
\left|T \backslash T_{1 n}\right|<\varepsilon,\left|T \backslash T_{2 n}\right|<\varepsilon \quad \text { and }\left|T_{1 n} \cap T_{2 n}\right| \geq \\
\left|T_{1 n}\right|-\left|T \backslash T_{2 n}\right|=|T|-\left|T \backslash T_{1 n}\right|-\left|T \backslash T_{2 n}\right| \geq b-2 \varepsilon .
\end{array}
$$

So, if $\varepsilon \in\left(0, \frac{b}{2}\right)$ then from (4) we see that $\left|T_{1 n} \cap T_{2 n}\right|>0$, which implies that $T_{1 n} \cap T_{2 n} \neq \emptyset$. If $t \in T_{1 n} \cap T_{2 n}$, then

$$
\frac{\left|u_{n}(t)\right|}{\left\|u_{n}\right\|} \geq \frac{\left|u_{n}^{0}(t)\right|}{\left\|u_{n}\right\|}-\frac{\left|\widehat{u}_{n}(t)+\bar{u}_{n}(t)\right|}{\left\|u_{n}\right\|} \geq \mu_{1} \frac{\left\|u_{n}^{0}\right\|}{\left\|u_{n}\right\|}-\mu_{2} \frac{\left\|\widehat{u}_{n}+\bar{u}_{n}\right\|}{\left\|u_{n}\right\|} .
$$

If $t \in T_{2 n} \backslash T_{1 n}$, then

$$
\frac{\left|u_{n}(t)\right|}{\left\|u_{n}\right\|} \leq \frac{\left|u_{n}^{0}(t)\right|}{\left\|u_{n}\right\|}+\frac{\left|\widehat{u}_{n}(t)+\bar{u}_{n}(t)\right|}{\left\|u_{n}\right\|}<\mu_{1} \frac{\left\|u_{n}^{0}\right\|}{\left\|u_{n}\right\|}+\mu_{2} \frac{\left\|\widehat{u}_{n}+\bar{u}_{n}\right\|}{\left\|u_{n}\right\|} .
$$

Since $\theta_{0} \in L^{\infty}(T)$ and $y_{n}=\frac{\left|u_{n}\right|}{\left\|u_{n}\right\|}$ satisfies $\left\|y_{n}\right\|=1$, then we can find $c_{1}>0$ such that

$$
\int_{0}^{b}\left(\frac{\left|u_{n}(t)\right|}{\left\|u_{n}\right\|}\right)^{2 \beta} d t \leq c_{1}
$$

By virtue of hypothesis $H(\mathrm{v})$, for the chosen $\varepsilon>0$, we can find $\delta=\delta(\varepsilon)>0$ such that

$$
f_{0}(t, x) x \leq\left(\theta_{0}(t)+\mu_{1}^{2 \beta} \varepsilon\right)|x|^{2 \beta} \quad \text { for a.a. } t \in T, \text { all }|x| \leq \delta .
$$

By hypothesis $\left\|u_{n}\right\| \rightarrow 0$. From the continuous (in fact compact) embedding of $W$ into $C(T)$, we have $\left\|u_{n}\right\|_{\infty} \rightarrow 0$ as $n \rightarrow \infty$. So, we can find $n_{0}=n_{0}(\delta) \geq 1$ such that $\left\|u_{n}\right\|_{\infty} \leq \delta$ for all $n \geq n_{0}$. Therefore

$$
f_{0}\left(t, u_{n}(t)\right) u_{n}(t) \leq\left(\theta_{0}(t)+\mu_{1}^{2 \beta} \varepsilon\right)\left|u_{n}(t)\right|^{2 \beta} \quad \text { for a.a. } t \in T \text {, all } n \geq n_{0} .
$$


Now, we evaluate the $\int_{0}^{b} \frac{f_{0}\left(t, u_{n}\right) u_{n}}{\left\|u_{n}\right\|^{2 \beta}} d t$ for $n \geq n_{0}$ by splitting it into two integrals. To this end, we recall that $\theta_{0} \leq 0$ a.e. on $T$. Then, using (4), (5) and (8) we obtain

$$
\begin{aligned}
& \int_{T_{1 n} \cap T_{2 n}} \frac{f_{0}\left(t, u_{n}\right) u_{n}}{\left\|u_{n}\right\|^{2 \beta}} d t \\
& \leq \int_{T_{1 n} \cap T_{2 n}}\left(\theta_{0}(t)+\mu_{1}^{2 \beta} \varepsilon\right)\left(\frac{\left|u_{n}(t)\right|}{\left\|u_{n}\right\|}\right)^{2 \beta} d t \\
& \leq\left[\frac{\mu_{1}^{2 \beta}}{2^{2 \beta-1}}\left(\frac{\left\|u_{n}^{0}\right\|}{\left\|u_{n}\right\|}\right)^{2 \beta}-\mu_{2}^{2 \beta}\left(\frac{\left\|\widehat{u}_{n}+\bar{u}_{n}\right\|}{\left\|u_{n}\right\|}\right)^{2 \beta}\right] \int_{T_{1 n} \cap T_{2 n}} \theta_{0} d t \\
& +\mu_{1}^{2 \beta} \varepsilon \int_{T_{1 n} \cap T_{2 n}}\left(\frac{\left|u_{n}(t)\right|}{\left\|u_{n}\right\|}\right)^{2 \beta} d t \\
& \leq\left[\frac{\mu_{1}^{2 \beta}}{2^{2 \beta-1}}\left(\frac{\left\|u_{n}^{0}\right\|}{\left\|u_{n}\right\|}\right)^{2 \beta}-\mu_{2}^{2 \beta}\left(\frac{\left\|\widehat{u}_{n}+\bar{u}_{n}\right\|}{\left\|u_{n}\right\|}\right)^{2 \beta}\right] \int_{0}^{b} \theta_{0} d t \\
& +\frac{\mu_{1}^{2 \beta}}{2^{2 \beta-1}}\left(\frac{\left\|u_{n}^{0}\right\|}{\left\|u_{n}\right\|}\right)^{2 \beta} \int_{T \backslash\left(T_{1 n} \cap T_{2 n}\right)}-\theta_{0} d t+\mu_{1}^{2 \beta} \varepsilon \int_{T_{1 n} \cap T_{2 n}}\left(\frac{\left|u_{n}(t)\right|}{\left\|u_{n}\right\|}\right)^{2 \beta} d t \\
& \leq\left[\frac{\mu_{1}^{2 \beta}}{2^{2 \beta-1}}\left(\frac{\left\|u_{n}^{0}\right\|}{\left\|u_{n}\right\|}\right)^{2 \beta}-\mu_{2}^{2 \beta}\left(\frac{\left\|\widehat{u}_{n}+\bar{u}_{n}\right\|}{\left\|u_{n}\right\|}\right)^{2 \beta}\right] \int_{0}^{b} \theta_{0} d t \\
& +\frac{\mu_{1}^{2 \beta}}{2^{2 \beta-1}}\left\|\theta_{0}\right\|_{\infty} 2 \varepsilon+\mu_{1}^{2 \beta} \varepsilon \int_{T_{1 n} \cap T_{2 n}}\left(\frac{\left|u_{n}(t)\right|}{\left\|u_{n}\right\|}\right)^{2 \beta} d t
\end{aligned}
$$

For the evaluation of the second integral we simply use (8):

$$
\int_{T \backslash\left(T_{1 n} \cap T_{2 n}\right)} \frac{f_{0}\left(t, u_{n}\right) u_{n}}{\left\|u_{n}\right\|^{2 \beta}} d t \leq \mu_{1}^{2 \beta} \varepsilon \int_{T \backslash\left(T_{1 n} \cap T_{2 n}\right)}\left(\frac{\left|u_{n}(t)\right|}{\left\|u_{n}\right\|}\right)^{2 \beta} d t
$$

Therefore, finally for $n \geq n_{0}$, owing to (7), and adding (9) and (10) we have

$$
\begin{aligned}
& \int_{0}^{b} \frac{f_{0}\left(t, u_{n}\right) u_{n}}{\left\|u_{n}\right\|^{2 \beta}} d t \leq\left[\frac{\mu_{1}^{2 \beta}}{2^{2 \beta-1}}\left(\frac{\left\|u_{n}^{0}\right\|}{\left\|u_{n}\right\|}\right)^{2 \beta}-\mu_{2}^{2 \beta}\left(\frac{\left\|\widehat{u}_{n}+\bar{u}_{n}\right\|}{\left\|u_{n}\right\|}\right)^{2 \beta}\right] \int_{0}^{b} \theta_{0} d t \\
& +\frac{\mu_{1}^{2 \beta}}{2^{2(\beta-1)}}\left\|\theta_{0}\right\|_{\infty} \varepsilon+\mu_{1}^{2 \beta} \varepsilon \int_{0}^{b}\left(\frac{\left|u_{n}(t)\right|}{\left\|u_{n}\right\|}\right)^{2 \beta} d t \\
& \leq\left[\frac{\mu_{1}^{2 \beta}}{2^{2 \beta-1}}\left(\frac{\left\|u_{n}^{0}\right\|}{\left\|u_{n}\right\|}\right)^{2 \beta}-\mu_{2}^{2 \beta}\left(\frac{\left\|\widehat{u}_{n}+\bar{u}_{n}\right\|}{\left\|u_{n}\right\|}\right)^{2 \beta}\right] \\
& \times \int_{0}^{b} \theta_{0} d t+\frac{\mu_{1}^{2 \beta} \varepsilon}{2^{2 \beta-2}}\left(\left\|\theta_{0}\right\|_{\infty}+c_{1} 2^{2 \beta-2}\right)
\end{aligned}
$$

In (11) we pass to the limit as $n \rightarrow \infty$ and use the fact that by hypothesis

$$
\frac{\left\|u_{n}^{0}\right\|}{\left\|u_{n}\right\|} \rightarrow 1 \text { and } \frac{\left\|\widehat{u}_{n}+\bar{u}_{n}\right\|}{\left\|u_{n}\right\|} \rightarrow 0 \text { as } n \rightarrow \infty .
$$


We obtain

$$
\limsup _{n \rightarrow \infty} \int_{0}^{b} \frac{f_{0}\left(t, u_{n}\right) u_{n}}{\left\|u_{n}\right\|^{2 \beta}} d t \leq \frac{\mu_{1}^{2 \beta}}{2^{2 \beta-1}} \int_{0}^{b} \theta_{0} d t+\frac{\mu_{1}^{2 \beta} \varepsilon}{2^{2 \beta-2}}\left(\left\|\theta_{0}\right\|_{\infty}+c_{1} 2^{2 \beta-2}\right) .
$$

Recall that $\varepsilon>0$ was arbitrary. So, choosing $\varepsilon \in(0,1)$ small we obtain

$$
\limsup _{n \rightarrow \infty} \int_{0}^{b} \frac{f_{0}\left(t, u_{n}\right) u_{n}}{\left\|u_{n}\right\|^{2 \beta}} d t<0 .
$$

We have a similar result for $f_{\infty}(t, x)$.

Proposition 3.2. If $\left\{u_{n}\right\}_{n \geq 1} \subseteq W$ is a sequence such that $\left\|u_{n}\right\| \rightarrow+\infty$ and $\frac{\widehat{u}_{n}+\bar{u}_{n}}{\left\|u_{n}\right\|} \rightarrow 0$ in $W$, then $\lim \sup _{n \rightarrow \infty} \int_{0}^{b} \frac{f_{\infty}\left(t, u_{n}\right) u_{n}}{\left\|u_{n}\right\|^{2 \alpha}} d t<0$.

Proof. For this proposition, we consider the following orthogonal direct sum decomposition

$$
W=\bar{W}_{m-1} \oplus E\left(\lambda_{m}\right) \oplus \widehat{W}_{m+1} .
$$

Again from Bartolo et al. [3], we know that given $\varepsilon>0$, we can find $\mu_{1}(\varepsilon), \mu_{2}(\varepsilon)>0$ such that

$$
\begin{gathered}
\left|\left\{t \in T:\left|u^{0}(t)\right|<\mu_{1}\left\|u^{0}\right\|\right\}\right|<\varepsilon \text { for all } u^{0} \in E\left(\lambda_{m}\right) \backslash\{0\} \text { and } \\
\qquad\left\{t \in T:|\widehat{u}(t)+\bar{u}(t)|>\mu_{2}\|\widehat{u}+\bar{u}\|\right\} \mid \\
<\mu_{1}^{2 \alpha} \varepsilon \text { for all } \widehat{u} \in \widehat{W}_{m+1}, \bar{u} \in \bar{W}_{m-1}, \widehat{u}+\bar{u} \neq 0 .
\end{gathered}
$$

For every $n \geq 1$, we consider the sets

$$
\begin{aligned}
& I_{1 n}=\left\{t \in T:\left|u_{n}^{0}(t)\right| \geq \mu_{1}\left\|u_{n}^{0}\right\|\right\} \quad \text { and } \\
& I_{2 n}=\left\{t \in T:\left|\widehat{u}_{n}(t)+\bar{u}_{n}(t)\right| \leq \mu_{2}\left\|\widehat{u}_{n}+\bar{u}_{n}\right\|\right\} .
\end{aligned}
$$

We have

$$
\left|T \backslash I_{1 n}\right|<\varepsilon,\left|T \backslash I_{2 n}\right|<\varepsilon \quad \text { and } \quad\left|I_{1 n} \cap I_{2 n}\right| \geq|T|-2 \varepsilon=b-2 \varepsilon .
$$

So, if we choose $\varepsilon \in\left(0, \frac{b}{2}\right)$ then from (12) we see that $\left|I_{1 n} \cap I_{2 n}\right| \neq \emptyset$. For $t \in I_{1 n} \cap I_{2 n}$, we have

$$
\frac{\left|u_{n}(t)\right|}{\left\|u_{n}\right\|} \geq \mu_{1} \frac{\left\|u_{n}^{0}\right\|}{\left\|u_{n}\right\|}-\mu_{2} \frac{\left\|\widehat{u}_{n}+\bar{u}_{n}\right\|}{\left\|u_{n}\right\|} .
$$

For $t \in I_{2 n} \backslash I_{1 n}$, we have

$$
\frac{\left|u_{n}(t)\right|}{\left\|u_{n}\right\|} \leq \mu_{1} \frac{\left\|u_{n}^{0}\right\|}{\left\|u_{n}\right\|}+\mu_{2} \frac{\left\|\widehat{u}_{n}+\bar{u}_{n}\right\|}{\left\|u_{n}\right\|} .
$$

By virtue of hypothesis $H$ (iii), (iv), given $\varepsilon>0$, we can find $a_{\varepsilon} \in L^{1}(T)_{+}$such that

$$
f_{\infty}(t, x) x \leq\left(\theta_{\infty}(t)+\mu_{1}^{2 \alpha} \varepsilon\right)|x|^{2 \alpha}+a_{\varepsilon}(t) \text { for a.a. } t \in T, \text { all } x \in \mathbb{R} .
$$

Now, we can find $c_{2}>0$ such that:

$$
\int_{0}^{b}\left(\frac{\left|u_{n}(t)\right|}{\left\|u_{n}\right\|}\right)^{2 \alpha} d t \leq c_{2}
$$


Then, arguing as for (9) and (10), recalling that $\theta_{\infty} \leq 0$ and using firstly (13) and (15), then only (15), we obtain

$$
\begin{aligned}
& \int_{I_{1 n} \cap I_{2 n}} \frac{f_{\infty}\left(t, u_{n}\right) u_{n}}{\left\|u_{n}\right\|^{2 \alpha}} d t \\
& \leq\left[\frac{\mu_{1}^{2 \alpha}}{c_{3}}\left(\frac{\left\|u_{n}^{0}\right\|}{\left\|u_{n}\right\|}\right)^{2 \alpha}-\mu_{2}^{2 \alpha}\left(\frac{\left\|\widehat{u}_{n}+\bar{u}_{n}\right\|}{\left\|u_{n}\right\|}\right)^{2 \alpha}\right] \int_{0}^{b} \theta_{\infty} d t \\
& \quad+\frac{\mu_{1}^{2 \alpha}}{c_{3}}\left(\frac{\left\|u_{n}^{0}\right\|}{\left\|u_{n}\right\|}\right)^{2 \alpha} \int_{T \backslash I_{1 n} \cap I_{2 n}}-\theta_{\infty} d t \\
& \quad+\mu_{1}^{2 \alpha} \varepsilon \int_{I_{1 n} \cap I_{2 n}}\left(\frac{\left|u_{n}(t)\right|}{\left\|u_{n}\right\|}\right)^{2 \alpha} d t+\int_{I_{1 n} \cap I_{2 n}} \frac{a_{\varepsilon}(t)}{\left\|u_{n}\right\|^{2 \alpha}} d t
\end{aligned}
$$

for some $c_{3} \geq 1$ and

$$
\begin{aligned}
& \int_{T \backslash\left(I_{1 n} \cap I_{2 n}\right)} \frac{f_{\infty}\left(t, u_{n}\right) u_{n}}{\left\|u_{n}\right\|^{2 \alpha}} d t \leq \mu_{1}^{2 \alpha} \varepsilon \int_{T \backslash\left(I_{1 n} \cap I_{2 n}\right)}\left(\frac{\left|u_{n}(t)\right|}{\left\|u_{n}\right\|}\right)^{2 \alpha} d t \\
& \quad+\int_{T \backslash\left(I_{1 n} \cap I_{2 n}\right)} \frac{a_{\varepsilon}(t)}{\left\|u_{n}\right\|^{2 \alpha}} d t .
\end{aligned}
$$

Adding (17) and (18) and using (12) and (16), we have

$$
\begin{aligned}
\int_{0}^{b} & \frac{f_{\infty}\left(t, u_{n}\right) u_{n}}{\left\|u_{n}\right\|^{2 \alpha}} d t \\
\leq & {\left[\frac{\mu_{1}^{2 \alpha}}{c_{3}}\left(\frac{\left\|u_{n}^{0}\right\|}{\left\|u_{n}\right\|}\right)^{2 \alpha}-\mu_{2}^{2 \alpha}\left(\frac{\left\|\widehat{u}_{n}+\bar{u}_{n}\right\|}{\left\|u_{n}\right\|}\right)^{2 \alpha}\right] \int_{0}^{b} \theta_{\infty} d t+\frac{\mu_{1}^{2 \alpha}}{c_{3}}\left(\frac{\left\|u_{n}^{0}\right\|}{\left\|u_{n}\right\|}\right)^{2 \alpha} } \\
& \times \int_{T \backslash I_{1} \cap I_{2 n}}-\theta_{\infty} d t+\mu_{1}^{2 \alpha} \varepsilon \int_{0}^{b}\left(\frac{\left|u_{n}(t)\right|}{\left\|u_{n}\right\|}\right)^{2 \alpha} d t+\int_{0}^{b} \frac{a_{\varepsilon}(t)}{\left\|u_{n}\right\|^{2 \alpha}} d t \\
\leq & {\left[\frac{\mu_{1}^{2 \alpha}}{c_{3}}\left(\frac{\left\|u_{n}^{0}\right\|}{\left\|u_{n}\right\|}\right)^{2 \alpha}-\mu_{2}^{2 \alpha}\left(\frac{\left\|\widehat{u}_{n}+\bar{u}_{n}\right\|}{\left\|u_{n}\right\|}\right)^{2 \alpha}\right] \int_{0}^{b} \theta_{\infty} d t } \\
& +\frac{\mu_{1}^{2 \alpha}}{c_{3}}\left\|\theta_{\infty}\right\|_{\infty} 2 \varepsilon+\mu_{1}^{2 \alpha} \varepsilon c_{2}+\frac{\left\|a_{\varepsilon}\right\|_{1}}{\left\|u_{n}\right\|^{2 \alpha}} .
\end{aligned}
$$

In (19) we pass to the limit as $n \rightarrow \infty$ and use the fact that

$$
\left\|u_{n}\right\| \rightarrow+\infty, \frac{\left\|u_{n}^{0}\right\|}{\left\|u_{n}\right\|} \rightarrow 1 \text { and } \frac{\left\|\widehat{u}_{n}+\bar{u}_{n}\right\|}{\left\|u_{n}\right\|} \rightarrow 0 .
$$

So, in the limit we have

$$
\limsup _{n \rightarrow \infty} \int_{0}^{b} \frac{f_{\infty}\left(t, u_{n}\right) u_{n}}{\left\|u_{n}\right\|^{2 \alpha}} d t \leq \frac{\mu_{1}^{2 \alpha}}{c_{3}} \int_{0}^{b} \theta_{\infty} d t+\frac{\varepsilon \mu_{1}^{2 \alpha}}{c_{3}}\left(2\left\|\theta_{\infty}\right\|_{\infty}+c_{2} c_{3}\right) .
$$

Recall that $\int_{0}^{b} \theta_{\infty} d t<0$. So choosing $\varepsilon>0$ small we obtain

$$
\limsup _{n \rightarrow \infty} \int_{0}^{b} \frac{f_{\infty}\left(t, u_{n}\right) u_{n}}{\left\|u_{n}\right\|^{2 \alpha}} d t<0 .
$$


Let $R>0$ and $\eta, \alpha \in(0,1)$ ( $\alpha$ as in hypothesis $H(\mathrm{iv})$ ). We introduce the set

$$
C_{\infty}(R, \eta, \alpha)=\left\{u \in W:\|u\| \geq R,\|\widehat{u}+\bar{u}\| \leq \eta\|u\|^{\alpha}\right\} .
$$

Proposition 3.3. If hypotheses $H$ hold, then there exist $R>0, \eta \in(0,1)$ and $\delta_{0}>0$ such that $\left\langle\varphi^{\prime}(u), u^{0}\right\rangle \geq \delta_{0}$ for all $u \in C_{\infty}(R, \eta, \alpha)$.

Proof. We proceed by contradiction. Assuming that the proposition is not true, then for every $n \geq 1$ and $\eta=\delta_{0}=\frac{1}{n}$, we can find $u_{n} \in W$ such that

$$
\left\|u_{n}\right\| \geq n,\left\|\widehat{u}_{n}+\bar{u}_{n}\right\| \leq \frac{1}{n}\left\|u_{n}\right\|^{\alpha} \quad \text { and } \quad\left\langle\varphi^{\prime}\left(u_{n}\right), u_{n}^{0}\right\rangle<\frac{1}{n} \text { for all } n \geq 1 .
$$

From (20) we have

$$
\left\|u_{n}\right\| \rightarrow \infty, \frac{\left\|\widehat{u}_{n}+\bar{u}_{n}\right\|}{\left\|u_{n}\right\|^{\alpha}} \rightarrow 0 \text { and since } \alpha \in(0,1) \text {, also } \frac{\left\|\widehat{u}_{n}+\bar{u}_{n}\right\|}{\left\|u_{n}\right\|} \rightarrow 0 \text {. }
$$

Moreover, exploiting the orthogonality of the component spaces in (20), we have

$$
\begin{aligned}
\left\langle\varphi^{\prime}\left(u_{n}\right), u_{n}^{0}\right\rangle & =\left\langle A\left(u_{n}\right), u_{n}^{0}\right\rangle-\lambda_{m}\left\|u_{n}^{0}\right\|_{2}^{2}-\int_{0}^{b} f_{\infty}\left(t, u_{n}\right) u_{n}^{0} d t \\
& =\left\|\left(u_{n}^{0}\right)^{\prime}\right\|_{2}^{2}-\lambda_{m}\left\|u_{n}^{0}\right\|_{2}^{2}-\int_{0}^{b} f_{\infty}\left(t, u_{n}\right) u_{n}^{0} d t \\
& =-\int_{0}^{b} f_{\infty}\left(t, u_{n}\right) u_{n}^{0} d t<\frac{1}{n}
\end{aligned}
$$

SO

$$
\liminf _{n \rightarrow \infty} \frac{1}{\left\|u_{n}\right\|^{2 \alpha}} \int_{0}^{b} f_{\infty}\left(t, u_{n}\right) u_{n}^{0} d t \geq 0 .
$$

By virtue of hypotheses $H$ (iii), (iv), given $\varepsilon>0$, we can find $\widehat{a}_{\varepsilon} \in L^{1}(T)_{+}$such that

$$
\left.\left|f_{\infty}(t, x) \leq \varepsilon\right| x\right|^{\alpha}+\widehat{a}_{\varepsilon}(t) \quad \text { for a.a. } t \in T \text {, all } x \in \mathbb{R} .
$$

Let $c_{0}$ be the constant of the embedding of $W$ in $C(T)$. From (23) we have

$$
\begin{aligned}
& \left|\int_{0}^{b} \frac{f_{\infty}\left(t, u_{n}(t)\right)\left(\widehat{u}_{n}(t)+\bar{u}_{n}(t)\right)}{\left\|u_{n}\right\|^{2 \alpha}} d t\right| \\
& \quad \leq \int_{0}^{b} \frac{\left(\varepsilon\left|u_{n}(t)\right|^{\alpha}+\widehat{a}_{\varepsilon}(t)\right)\left|\widehat{u}_{n}(t)+\bar{u}_{n}(t)\right|}{\left\|u_{n}\right\|^{2 \alpha}} d t \\
& \quad \leq b \varepsilon\left(\frac{c_{0}\left\|u_{n}\right\|}{\left\|u_{n}\right\|}\right)^{\alpha} \cdot c_{0} \frac{\left\|\widehat{u}_{n}+\bar{u}_{n}\right\|}{\left\|u_{n}\right\|^{\alpha}} d t+c_{0}\left\|\widehat{a}_{\varepsilon}\right\|_{1} \frac{\left\|\widehat{u}_{n}+\bar{u}_{n}\right\|}{\left\|u_{n}\right\|^{2 \alpha}} \\
& \quad \leq\left(b \varepsilon c_{0}^{1+\alpha}+c_{0}\left\|\widehat{a}_{\varepsilon}\right\|_{1}\right) \frac{\left\|\widehat{u}_{n}+\bar{u}_{n}\right\|}{\left\|u_{n}\right\|^{\alpha}} \text { for all } n \geq 1,
\end{aligned}
$$

SO

$$
\lim _{n \rightarrow \infty} \int_{0}^{b} \frac{f_{\infty}\left(t, u_{n}(t)\right)\left(\widehat{u}_{n}(t)+\bar{u}_{n}(t)\right)}{\left\|u_{n}\right\|^{2 \alpha}} d t=0
$$


From this last equality and Proposition 3.2 it follows that

$$
\begin{aligned}
& \limsup _{n \rightarrow \infty} \int_{0}^{b} \frac{f_{\infty}\left(t, u_{n}(t)\right) u_{n}^{0}(t)}{\left\|u_{n}\right\|^{2 \alpha}} d t \\
& \quad=\limsup _{n \rightarrow \infty}\left[\int_{0}^{b} \frac{f_{\infty}\left(t, u_{n}(t)\right) u_{n}(t)}{\left\|u_{n}\right\|^{2 \alpha}} d t-\int_{0}^{b} \frac{f_{\infty}\left(t, u_{n}(t)\right)\left(\widehat{u}_{n}(t)+\bar{u}_{n}(t)\right)}{\left\|u_{n}\right\|^{2 \alpha}} d t\right] \\
& \quad \leq \limsup _{n \rightarrow \infty} \int_{0}^{b} \frac{f_{\infty}\left(t, u_{n}(t)\right) u_{n}(t)}{\left\|u_{n}\right\|^{2 \alpha}} d t<0
\end{aligned}
$$

Comparing (22) and (25), we reach a contradiction. This proves the proposition.

Now we are ready to prove that the energy functional $\varphi$ satisfies the C-condition.

Proposition 3.4. If hypotheses $H$ hold, then the functional $\varphi$ satisfies the C-condition.

Proof. Let $\left\{u_{n}\right\}_{n \geq 1} \subseteq W$ be a sequence such that $\left\{\varphi\left(u_{n}\right)\right\}_{n \geq 1} \subseteq \mathbb{R}$ is bounded and

$$
\left(1+\left\|u_{n}\right\|\right) \varphi^{\prime}\left(u_{n}\right) \rightarrow 0 \text { in } W^{*} \text { as } n \rightarrow \infty .
$$

We will show that the sequence $\left\{u_{n}\right\}_{n \geq 1} \subseteq W$ is bounded. If this is not the case, by passing to a suitable subsequence if necessary, we may assume that $\left\|u_{n}\right\| \rightarrow \infty$. From (26) we have

$$
\left|\left\langle\varphi^{\prime}\left(u_{n}\right), h\right\rangle\right| \leq \frac{\varepsilon_{n}\|h\|}{1+\left\|u_{n}\right\|} \quad \text { for all } h \in W, \text { with } \varepsilon_{n} \rightarrow 0^{+} .
$$

In (27) we choose $h=\widehat{u}_{n} \in W$. Exploiting the orthogonality of the component spaces, we have

$$
\begin{aligned}
\left\langle\varphi^{\prime}\left(u_{n}\right), \widehat{u}_{n}\right\rangle & =\left\langle A\left(u_{n}\right), \widehat{u}_{n}\right\rangle-\lambda_{m} \int_{0}^{b} u_{n} \widehat{u}_{n} d t-\int_{0}^{b} f_{\infty}\left(t, u_{n}\right) \widehat{u}_{n} d t \\
& =\left\|\widehat{u}_{n}^{\prime}\right\|_{2}^{2}-\lambda_{m}\left\|\widehat{u}_{n}\right\|_{2}^{2}-\int_{0}^{b} f_{\infty}\left(t, u_{n}\right) \widehat{u}_{n} d t \leq \varepsilon_{n} \quad \text { for all } n \geq 1,
\end{aligned}
$$

and from this, using (23) and bearing in mind that $\widehat{u}_{n} \in \widehat{W}_{m+1}$, we can find a number $\widehat{\xi}_{0}>0$ such that

$\widehat{\xi}_{0}\left\|\widehat{u}_{n}\right\|^{2} \leq \varepsilon_{n}+\int_{0}^{b} f_{\infty}\left(t, u_{n}\right) \widehat{u}_{n} d t \leq \varepsilon_{n}+\int_{0}^{b}\left(\varepsilon\left|u_{n}\right|^{\alpha}+\widehat{a}_{\varepsilon}(t)\right) \widehat{u}_{n} d t \quad$ for all $n \geq 1$,

so, for all $n \geq 1$, we have

$$
\widehat{\xi}_{0}\left\|\widehat{u}_{n}\right\|^{2} \leq \varepsilon_{n}+b \varepsilon c_{0}^{1+\alpha}\left\|u_{n}\right\|^{\alpha}\left\|\widehat{u}_{n}\right\|+c_{0}\left\|\widehat{a}_{\varepsilon}\right\|_{1}\left\|\widehat{u}_{n}\right\|,
$$

hence

$\widehat{\xi}_{0}\left(\frac{\left\|\widehat{u}_{n}\right\|}{\left\|u_{n}\right\|^{\alpha}}\right)^{2} \leq \frac{\varepsilon_{n}}{\left\|u_{n}\right\|^{2 \alpha}}+\left(b \varepsilon c_{0}^{1+\alpha}+\frac{c_{0}\left\|\widehat{a}_{\varepsilon}\right\|_{1}}{\left\|u_{n}\right\|^{\alpha}}\right) \frac{\left\|\widehat{u}_{n}\right\|}{\left\|u_{n}\right\|^{\alpha}} \quad$ for all $n \geq 1$,

and $\left\{\sigma_{n}=\frac{\left\|\widehat{u}_{n}\right\|}{\left\|u_{n}\right\|^{\alpha}}\right\}_{n \geq 1}$ is bounded. 
We may assume that $\sigma_{n} \rightarrow \sigma \geq 0$ as $n \rightarrow \infty$. So, if in (28) we pass to the limit as $n \rightarrow \infty$, then

$$
\widehat{\xi}_{0} \sigma^{2} \leq b \varepsilon c_{0}^{1+\alpha} \sigma .
$$

Since $\varepsilon>0$ was arbitrary, we let $\varepsilon \rightarrow 0^{+}$to conclude that $\sigma=0$. Therefore

$$
\frac{\left\|\widehat{u}_{n}\right\|}{\left\|u_{n}\right\|^{\alpha}} \rightarrow 0 \text { in } W \text { as } n \rightarrow \infty \text {. }
$$

Next, in (27) we choose $h=-\bar{u}_{n} \in \bar{W}_{m-1}$. Then we can find $\widehat{\xi}_{1}>0$ such that

$$
\widehat{\xi}_{1}\left\|\bar{u}_{n}\right\|^{2} \leq \varepsilon_{n}+\int_{0}^{b}\left|f_{\infty}\left(t, u_{n}\right)\right|\left|\bar{u}_{n}\right| d t \text { for all } n \geq 1,
$$

hence (see $(23))$, for all $n \geq 1$ we have

$$
\widehat{\xi}_{1}\left(\frac{\left\|\bar{u}_{n}\right\|}{\left\|u_{n}\right\|^{\alpha}}\right)^{2} \leq \frac{\varepsilon_{n}}{\left\|u_{n}\right\|^{2 \alpha}}+\left(b \varepsilon c_{0}^{1+\alpha}+\frac{c_{0}\left\|\widehat{a}_{\varepsilon}\right\|_{1}}{\left\|u_{n}\right\|^{\alpha}}\right) \frac{\left\|\bar{u}_{n}\right\|}{\left\|u_{n}\right\|^{\alpha}},
$$

and, as above we deduce that $\frac{\left\|\bar{u}_{n}\right\|}{\left\|u_{n}\right\|^{\alpha}} \rightarrow 0$ in $W$ as $n \rightarrow \infty$.

Let $R>0, \eta \in(0,1)$ and $\delta_{0}>0$ be as postulated in Proposition 3.3. Since $\left\|u_{n}\right\|$ is not bounded, from (29) and (30) it follows that $u_{n} \in C_{\infty}(R, \eta, \alpha)$ for all $n \geq n_{0}$ and so by virtue of Proposition 3.3

$$
\left\langle\varphi^{\prime}\left(u_{n}\right), u_{n}^{0}\right\rangle \geq \delta_{0} \quad \text { for all } n \geq n_{0} .
$$

On the other hand, if in (27) we choose $h=u_{n}^{0} \in E\left(\lambda_{m}\right)$, then

$$
\left\langle\varphi^{\prime}\left(u_{n}\right), u_{n}^{0}\right\rangle \leq \varepsilon_{n} \quad \text { for all } n \geq n_{0} \quad \text { and } \quad \varepsilon_{n} \rightarrow 0^{+} .
$$

Comparing (31) and (32), we reach a contradiction. This proves that $\left\{u_{n}\right\} \subseteq W$ is bounded. Hence we may assume that

$$
u_{n} \rightarrow u \text { in } W \text { and } u_{n} \rightarrow u \text { in } C(T) \text { as } n \rightarrow \infty .
$$

In (27) we choose $h=u_{n}-u \in W$. Then

$$
\left|\left\langle A\left(u_{n}\right), u_{n}-u\right\rangle-\int_{0}^{b} f\left(t, u_{n}\right)\left(u_{n}-u\right) d t\right| \leq \frac{\varepsilon_{n}\left\|u_{n}-u\right\|}{1+\left\|u_{n}\right\|} ;
$$

from this, using (33), we deduce that $\lim _{n \rightarrow \infty}\left\langle A\left(u_{n}\right), u_{n}-u\right\rangle=0$; since $A\left(u_{n}\right) \rightarrow A(u)$ in $W^{*}$, we have that $\left\|u_{n}^{\prime}\right\|_{2} \rightarrow\left\|u^{\prime}\right\|_{2}$. Finally, from the KadecKlee property of Hilbert spaces, we have $u_{n}^{\prime} \rightarrow u^{\prime}$ in $L^{2}(T)$. Hence $u_{n} \rightarrow u$ in $W$ and $\varphi$ satisfies the $C$-condition.

Next we will compute the critical groups of $\varphi$ at the origin and at infinity. First we consider the critical groups at the origin. For this purpose let $\beta>1$ be as in hypothesis $H(\mathrm{v})$ and introduce the following set

$C_{0}(\rho, \eta, \beta)=\left\{u \in W,:\|u\| \leq \rho,\|\widehat{u}+\bar{u}\| \leq \eta\|u\|^{\beta}\right\} \quad$ with $\rho>0, \eta \in(0,1)$.

Recall that since we are dealing with $\varphi$ near the origin, we use the following direct sum decomposition

$$
W=\bar{W}_{i-1} \oplus E\left(\lambda_{i}\right) \oplus \widehat{W}_{i+1} .
$$


Proposition 3.5. If hypotheses $H$ hold then $C_{k}(\varphi, 0) \equiv \delta_{k, d_{i}} Z$ for all $k \geq 0$, where $d_{i}=\operatorname{dim} \bar{W}_{i-1}$.

Proof. First we show that there exists $\rho>0$ and $\eta \in(0,1)$ such that

$$
\left\langle\varphi^{\prime}(u), u^{0}\right\rangle \geq 0 \quad \text { for all } u \in C_{0}(\rho, \eta, \beta) .
$$

We argue by contradiction. So, we may assume that for every $n \geq 1$ and $\rho=\eta=\frac{1}{n}$ we can find $u_{n} \in W$ such that

$$
\left\|u_{n}\right\| \leq \frac{1}{n},\left\|\widehat{u}_{n}+\bar{u}_{n}\right\| \leq \frac{1}{n}\left\|u_{n}\right\|^{\beta} \text { and }\left\langle\varphi^{\prime}\left(u_{n}\right), u_{n}^{0}\right\rangle<0 \quad \text { for all } n \geq 1 .
$$

From (35) we have

$$
\left\|u_{n}\right\| \rightarrow 0, \frac{\left\|\widehat{u}_{n}+\bar{u}_{n}\right\|}{\left\|u_{n}\right\|^{\beta}} \rightarrow 0 \quad \text { as } n \rightarrow \infty,
$$

and

$$
\begin{aligned}
\left\langle\varphi^{\prime}\left(u_{n}\right), u_{n}^{0}\right\rangle & =\left\langle A\left(u_{n}\right), u_{n}^{0}\right\rangle-\lambda_{i} \int_{0}^{b} u_{n} u_{n}^{0} d t-\int_{0}^{b} f_{0}\left(t, u_{n}\right) u_{n}^{0} d t \\
& =\left\|\left(u_{n}^{0}\right)^{\prime}\right\|_{2}^{2}-\lambda_{i}\left\|u_{n}^{0}\right\|_{2}^{2}-\int_{0}^{b} f_{0}\left(t, u_{n}\right) u_{n}^{0} d t \\
& =-\int_{0}^{b} f_{0}\left(t, u_{n}\right) u_{n}^{0} d t<0 \quad\left(\text { since } u_{n}^{0} \in E\left(\lambda_{i}\right)\right),
\end{aligned}
$$

SO

$$
\liminf _{n \rightarrow+\infty} \int_{0}^{b} \frac{f_{0}\left(t, u_{n}\right) u_{n}^{0}}{\left\|u_{n}\right\|^{2 \beta}} d t \geq 0 .
$$

Hypotheses $H$ (iii), (v), imply that given $\varepsilon>0$, we can find $\tilde{a}_{\varepsilon} \in L^{1}(T)_{+}$such that

$$
\left|f_{0}(t, x)\right| \leq \varepsilon|x|^{\beta}+\tilde{a}_{\varepsilon}(t)|x|^{\gamma} \text { for a.a. } t \in T \text {, all } x \in \mathbb{R} \text { with } \gamma>\beta .
$$

Then

$$
\begin{aligned}
& \left|\int_{0}^{b} \frac{f_{0}\left(t, u_{n}\right)\left(\widehat{u}_{n}+\bar{u}_{n}\right)}{\left\|u_{n}\right\|^{2 \beta}} d t\right| \\
& \quad \leq \int_{0}^{b} \frac{\left(\varepsilon\left|u_{n}\right|^{\beta}+\tilde{a}_{\varepsilon}(t)\left|u_{n}\right|^{\gamma}\right)\left|\widehat{u}_{n}+\bar{u}_{n}\right|}{\left\|u_{n}\right\|^{2 \beta}} d t \\
& \quad \leq\left(b \varepsilon c_{0}^{1+\beta}+c_{0}^{1+\gamma}\left\|\widehat{a}_{\varepsilon}\right\|_{1}\left\|u_{n}\right\|^{\gamma-\beta}\right) \frac{\left\|\widehat{u}_{n}+\bar{u}_{n}\right\|}{\left\|u_{n}\right\|^{\beta}} \quad \text { for all } n \geq 1,
\end{aligned}
$$

SO

$$
\lim _{n \rightarrow \infty} \int_{0}^{b} \frac{f_{0}\left(t, u_{n}\right)\left(\widehat{u}_{n}+\bar{u}_{n}\right)}{\left\|u_{n}\right\|^{2 \beta}} d t=0 .
$$

It follows that

$$
\liminf _{n \rightarrow \infty} \int_{0}^{b} \frac{f_{0}\left(t, u_{n}\right) u_{n}^{0}}{\left\|u_{n}\right\|^{2 \beta}} d t=\liminf _{n \rightarrow \infty} \int_{0}^{b} \frac{f_{0}\left(t, u_{n}\right) u_{n}}{\left\|u_{n}\right\|^{2 \beta}} d t \geq 0
$$

This last inequality contradicts Proposition 3.1. Therefore, we can find $\rho>0$ and $\eta \in(0,1)$ such that $(34)$ holds. 
Now let $\lambda \in\left(0, \lambda_{i}-\lambda_{i-1}\right)$ (recall $i \geq 1$, see $\left.H(\mathrm{v})\right)$ and consider the following homotopy

$$
h_{t}(u)=\varphi(u)+t \frac{\lambda}{2}\left\|u^{0}\right\|^{2} \quad \text { for all }(t, u) \in[0,1] \times W .
$$

Claim. There exists $r_{0}>0$ small such that $u \equiv 0$ is the only critical point of $\left\{h_{t}(\cdot)\right\}_{t \in[0,1]}$ in $\bar{B}_{r_{0}}(0)$.

To prove the Claim, we argue indirectly. So, suppose we can find $\left\{t_{n}\right\}_{n \geq 1} \subseteq[0,1] \backslash\{0\}$ and $\left\{u_{n}\right\}_{n \geq 1} \subseteq W$ such that

$t_{n} \rightarrow t \in[0,1],\left\|u_{n}\right\| \rightarrow 0 \quad$ and $\quad h_{t_{n}}^{\prime}\left(u_{n}\right)=\varphi^{\prime}\left(u_{n}\right)+t_{n} \lambda u_{n}^{0}=0 \forall n \geq 1$.

From the last equation in (40), we have

$$
\left\langle\varphi^{\prime}\left(u_{n}\right), u_{n}^{0}\right\rangle=-t_{n} \lambda\left\|u_{n}^{0}\right\|_{2}^{2} \quad \text { for all } n \geq 1 .
$$

Suppose that $u_{n}^{0} \equiv 0$ for all $n \geq n_{0} \geq 1$. Then, for $n \geq n_{0}$, we have

$$
A\left(u_{n}\right)-N\left(u_{n}\right)=\varphi^{\prime}\left(u_{n}\right)=0, \text { where } N(y)(\cdot)=f(\cdot, y(\cdot)) \forall y \in W .
$$

If we set $y_{n}=\frac{u_{n}}{\left\|u_{n}\right\|}, n \geq 1$, then we may assume that

$$
y_{n} \rightarrow y \text { in } W \text { and } y_{n} \rightarrow y \text { in } C(T) \text { as } n \rightarrow+\infty .
$$

From (42) we have

$$
A\left(y_{n}\right)=\frac{N\left(u_{n}\right)}{\left\|u_{n}\right\|} \quad \text { for all } n \geq n_{0} .
$$

Evidently $\left\{\frac{N\left(u_{n}\right)}{\left\|u_{n}\right\|}\right\}_{n \geq 1} \subseteq L^{1}(T)$ is uniformly integrable (see $H$ (iii) and (40)). So, by virtue of the Dunford-Pettis theorem, we may assume that $\frac{N\left(u_{n}\right)}{\left\|u_{n}\right\|} \rightarrow g$ in $L^{1}(T)$ as $n \rightarrow \infty$. Using hypothesis $H(\mathrm{v})$ and reasoning as in the proof of Proposition 5 of [14], we obtain $g=\lambda_{i} y$. Hence, if in (44) we pass to the limit as $n \rightarrow \infty$, and we take into account (43), then we obtain $A(y)=\lambda_{i} y$, that is

$$
-y^{\prime \prime}(t)=\lambda_{i} y(t) \text { a.e. on } T, y(0)=y(b), \quad y^{\prime}(0)=y^{\prime}(b),
$$

so $y \in E\left(\lambda_{i}\right)$. In fact, acting in (44) with $y_{n}-y$ and arguing as in the proof of Proposition 3.4, from (43) we deduce that $y_{n} \rightarrow y$ in $W,\|y\|=1$ and $y \in E\left(\lambda_{i}\right) \backslash\{0\}$. Bearing in mind the decomposition adopted for $W$, we can write $y=y^{0}$. Then $y_{n}^{0}=\frac{u_{n}^{0}}{\left\|u_{n}\right\|} \rightarrow y^{0} \neq 0$, which contradicts the hypothesis that $u_{n}^{0}=0$ for all $n \geq n_{0}$. Therefore by passing to a suitable subsequence if necessary, we may assume that $u_{n}^{0} \neq 0$ for all $n \geq 1$.

Let $\rho>0$ and $\eta \in(0,1)$ be as in (34). Suppose that $u_{n} \in C_{0}(\rho, \eta, \beta)$ for all $n \geq \hat{n}_{0}$. Then from (34) we have

$$
\left\langle\varphi^{\prime}\left(u_{n}\right), u_{n}^{0}\right\rangle \geq 0 \text { for all } n \geq \hat{n}_{0},
$$

which contradicts (41). Hence, by passing to a suitable subsequence if necessary, we may assume that $u_{n} \notin C_{0}(\rho, \eta, \beta)$ for all $n \geq 1$. Then

$$
\left\|\hat{u}_{n}+\bar{u}_{n}\right\|>\eta\left\|u_{n}\right\|^{\beta} \text { for all } n \geq 1 \text {. }
$$


From the orthogonality of the component spaces and (40), we have

$$
\begin{aligned}
0 & =\left\langle h_{t_{n}}^{\prime}\left(u_{n}\right), \hat{u}_{n}-\bar{u}_{n}\right\rangle=\left\langle\varphi^{\prime}\left(u_{n}\right), \hat{u}_{n}-\bar{u}_{n}\right\rangle \\
& =\left\|\hat{u}_{n}^{\prime}\right\|_{2}^{2}-\lambda_{i}\left\|\hat{u}_{n}\right\|_{2}^{2}-\left\|\bar{u}_{n}^{\prime}\right\|_{2}^{2}+\lambda_{i}\left\|\bar{u}_{n}\right\|_{2}^{2}-\int_{0}^{b} f_{0}\left(t, u_{n}\right)\left(\hat{u}_{n}-\bar{u}_{n}\right) d t \\
& \geq \hat{\xi}_{0}\left\|\hat{u}_{n}\right\|^{2}+\hat{\xi}_{1}\left\|\bar{u}_{n}\right\|^{2}-\int_{0}^{b} f_{0}\left(t, u_{n}\right)\left(\hat{u}_{n}-\bar{u}_{n}\right) d t \quad \text { for all } n \geq 1 .
\end{aligned}
$$

Now, owing to (38) and the fact that $\left\|\hat{u}_{n}-\bar{u}_{n}\right\|=\left\|\hat{u}_{n}+\bar{u}_{n}\right\|$, if we choose $c_{3}=\max \left\{c_{0}^{1+\beta}, c_{0}^{1+\gamma}\left\|\widehat{a}_{\varepsilon}\right\|_{1}\right\}$, then we obtain

$$
\begin{aligned}
& \hat{\xi}_{0}\left\|\hat{u}_{n}\right\|^{2}+\hat{\xi}_{1}\left\|\bar{u}_{n}\right\|^{2}-\int_{0}^{b} f_{0}\left(t, u_{n}\right)\left(\hat{u}_{n}-\bar{u}_{n}\right) d t \\
& \quad \geq \hat{\xi}_{0}\left\|\hat{u}_{n}\right\|^{2}+\hat{\xi}_{1}\left\|\bar{u}_{n}\right\|^{2}-c_{3}\left\|\hat{u}_{n}+\bar{u}_{n}\right\|\left(\varepsilon\left\|u_{n}\right\|^{\beta}+\left\|u_{n}\right\|^{\gamma}\right) \quad \text { for all } n \geq 1 .
\end{aligned}
$$

Finally, if we choose $\hat{\xi}_{2}=\min \left\{\hat{\xi}_{0}, \hat{\xi}_{1}\right\}$, use (45) and put together the previous inequalities, then we obtain

$$
\begin{aligned}
0 & \geq \hat{\xi}_{2}\left\|\hat{u}_{n}+\bar{u}_{n}\right\|^{2}-c_{3}\left\|\hat{u}_{n}+\bar{u}_{n}\right\|\left(\varepsilon\left\|u_{n}\right\|^{\beta}+\left\|u_{n}\right\|^{\gamma}\right) \\
& =\left\|\hat{u}_{n}+\bar{u}_{n}\right\|^{2}\left[\hat{\xi}_{2}-c_{3}\left(\frac{\varepsilon\left\|u_{n}\right\|^{\beta}}{\left\|\hat{u}_{n}+\bar{u}_{n}\right\|}+\frac{\left\|u_{n}\right\|^{\gamma}}{\left\|\hat{u}_{n}+\bar{u}_{n}\right\|}\right)\right] \\
& \geq\left\|\hat{u}_{n}+\bar{u}_{n}\right\|^{2}\left[\hat{\xi}_{2}-\frac{c_{3}}{\eta}\left(\varepsilon+\left\|u_{n}\right\|^{\gamma-\beta}\right)\right] .
\end{aligned}
$$

Since $\gamma>\beta$ and $\left\|u_{n}\right\| \rightarrow 0$, we can have $\left\|u_{n}\right\|^{\gamma-\beta}<\varepsilon$, hence (see (46)) $\hat{\xi}_{2} \leq \frac{2 \varepsilon c_{3}}{\eta}$. Choosing $\varepsilon>0$ small we obtain $\hat{\xi}_{2}>\frac{2 \varepsilon c_{3}}{\eta}$ and this leads to a contradiction. This proves the Claim.

The orthogonality of the component spaces, implies

$\left\langle h_{t}^{\prime}(u), \hat{u}\right\rangle=\left\langle\varphi^{\prime}(u), \hat{u}\right\rangle \quad$ and $\quad\left\langle h_{t}^{\prime}(u), \bar{u}\right\rangle=\left\langle\varphi^{\prime}(u), \bar{u}\right\rangle \quad$ for all $(t, u) \in[0,1] \times W$.

Using these facts and reasoning as in the proof of Proposition 3.4, we show that for all $t \in[0,1], h_{t}(\cdot)$ satisfies the C-condition. Then by virtue of the homotopy invariance of the critical groups (see $\left[6\right.$, p. 332]) and being $h_{0} \equiv \varphi$, we have

$$
C_{k}(\varphi, 0)=C_{k}\left(h_{1}, 0\right) \text { for all } k \geq 0 .
$$

Note that $h_{1}(u)=\varphi(u)+\frac{\lambda}{2}\left\|u^{0}\right\|^{2}$ for all $u \in W$. Clearly $h_{1} \in C^{2}(W)$ and $u=0$ is a critical point of $h_{1}$. We claim that $u=0$ is a nondegenerate critical point of $h_{1}$. To this end, let $y \in k e r h_{1}^{\prime \prime}(0)$. Then

$$
h_{1}^{\prime \prime}(0) y=\varphi^{\prime \prime}(0) y+\lambda y=0,
$$

hence

$$
A(y)-\lambda_{i} y-\left(f_{0}\right)_{x}^{\prime}(\cdot, 0) y+\lambda y=0 .
$$

Note that $f_{0}(t, 0)=0$ for a.a. $t \in T$ (see $H($ ii $\left.)\right)$, and $\frac{f_{0}(t, x)}{x}=\frac{f(t, x)}{x}-\lambda_{i}$, hence $\lim _{x \rightarrow 0} \frac{f_{0}(t, x)}{x}=0$ uniformly for a.a. $t \in T($ see $H(\mathrm{v}))$, so $\left(f_{0}\right)_{x}^{\prime}(t, 0)=0$ for a.a. $t \in T$. Using this in (48), we have $A(y)=\left(\lambda_{i}-\lambda\right) y$, hence

$$
-y^{\prime \prime}(t)=\left(\lambda_{i}-\lambda\right) y(t) \text { a.e. on } T, y(0)=y(b), y^{\prime}(0)=y^{\prime}(b) \text {. }
$$


Proposition 2 of Aizicovici et al. [1] and (49), imply that $y=0$ (recall that $\lambda \in\left(\lambda_{i}-\lambda_{i-1}\right)$. Hence $\operatorname{kerh}_{1}^{\prime \prime}(0)=\{0\}$ and so $u=0$ is a nondegenerate critical point of $h_{1}$ and its Morse index is $d_{i}=\operatorname{dim} \bar{W}_{i-1}$. From (47) and Mawhin and Willem [13, p. 188], we have

$$
C_{k}(\varphi, 0)=C_{k}\left(h_{1}, 0\right)=\delta_{k, d_{i}} Z \quad \text { for all } k \geq 0 .
$$

Next we will compute the critical groups of $\varphi$ at infinity. Now we are dealing with the orthogonal direct sum decomposition

$$
W=\bar{W}_{m-1} \oplus E\left(\lambda_{m}\right) \oplus \widehat{W}_{m+1} .
$$

Proposition 3.6. If hypotheses $H$ hold, then $C_{k}(\varphi, \infty) \equiv \delta_{k, d_{m}} \mathbb{Z}$ for all $k \geq 0$, where $d_{m}=\operatorname{dim} \bar{W}_{m-1}$.

Proof. In this case, we consider the following homotopy

$$
h_{t}(u)=\varphi(u)+\frac{t}{2}\left\|u^{0}\right\|^{2} \quad \text { for all }(t, u) \in[0,1] \times W .
$$

Note that for all $t \in[0,1], h_{t} \in C^{2}(W)$ and $h_{t}^{\prime}, \partial_{t} h_{t}$ are both locally Lipschitz.

Claim. There exists $a \in \mathbb{R}$ and $\delta>0$ such that if $h_{t}(u) \leq a$ then

$$
(1+\|u\|)\left\|h_{t}^{\prime}(u)\right\| \geq \delta \quad \text { for all } t \in[0,1] .
$$

$W e$ proceed by contradiction. Note that $(t, u) \rightarrow h_{t}(u)$ maps bounded sets to bounded ones. So, if the Claim is not true, then we can find $\left\{t_{n}\right\}_{n \geq 1} \subseteq[0,1]$ and $\left\{u_{n}\right\}_{n \geq 1} \subseteq W$ such that

$$
\begin{aligned}
& t_{n} \rightarrow t,\left\|u_{n}\right\| \rightarrow \infty, h_{t_{n}}\left(u_{n}\right) \rightarrow-\infty \text { and } \\
& \quad \times\left(1+\left\|u_{n}\right\|\right) h_{t_{n}}^{\prime}\left(u_{n}\right) \rightarrow 0 \text { in } W^{*} \text { as } n \rightarrow \infty .
\end{aligned}
$$

From the last convergence in (50), we have

SO

$$
\left|\left\langle h_{t_{n}}^{\prime}\left(u_{n}\right), v\right\rangle\right| \leq \frac{\varepsilon_{n}\|v\|}{1+\left\|u_{n}\right\|} \quad \text { for all } v \in W, \text { with } \varepsilon_{n} \rightarrow 0^{+},
$$

$$
\left|\left\langle A\left(u_{n}\right), v\right\rangle-\int_{0}^{b} N\left(u_{n}\right) v d t+t_{n} \int_{0}^{b} u_{n}^{0} v d t\right| \leq \frac{\varepsilon_{n}\|v\|}{1+\left\|u_{n}\right\|} \quad \text { for all } n \geq 1 .
$$

If we set $y_{n}=\frac{u_{n}}{\left\|u_{n}\right\|}, n \geq 1$, then we may assume that

$$
y_{n} \rightarrow y \text { in } W \quad \text { and } \quad y_{n} \rightarrow y \text { in } C(T) \text { as } n \rightarrow \infty .
$$

From (51) we have

$$
\left|\left\langle A\left(y_{n}\right), v\right\rangle-\int_{0}^{b} \frac{N\left(u_{n}\right)}{\left\|u_{n}\right\|} v d t+t_{n} \int_{0}^{b} y_{n}^{0} v d t\right| \leq \frac{\varepsilon_{n}\|v\|}{\left(1+\left\|u_{n}\right\|\right)\left\|u_{n}\right\|} \forall n \geq 1 .
$$

As before, using $H(\mathrm{v})$, the Dunford-Pettis theorem and reasoning as in [14], we have

$$
\frac{N\left(u_{n}\right)}{\left\|u_{n}\right\|} \rightarrow \lambda_{m} y \text { in } L^{1}(T)
$$


If in (53) we pass to the limit as $n \rightarrow \infty$, and use (52) and (54), then

$$
\langle A(y), v\rangle=\lambda_{m} \int_{0}^{b} y v d t-t \int_{0}^{b} y^{0} v d t \quad \text { for all } v \in W .
$$

Moreover, if in (53) we choose $v=y_{n}-y$ and pass to the limit as $n \rightarrow \infty$, then, bearing in mind (52) and (54), we obtain $\lim _{n \rightarrow \infty}\left\langle A\left(y_{n}\right), y_{n}-y\right\rangle=0$, hence

$$
y_{n} \rightarrow y \text { in } W \quad \text { and so } \quad\|y\|=1 .
$$

Next in (55), we choose first $v=\widehat{y} \in \widehat{W}_{m+1}$ and then $v=\bar{y} \in \bar{W}_{m-1}$. We obtain $\left\|\widehat{y}^{\prime}\right\|_{2}^{2}=\lambda_{m}\|\widehat{y}\|_{2}^{2}$ and $\left\|\bar{y}^{\prime}\right\|_{2}^{2}=\lambda_{m}\|\bar{y}\|_{2}^{2}$, and so $\widehat{y}=\bar{y}=0$. Hence

$$
y=y^{0} \in E\left(\lambda_{m}\right) \backslash\{0\} .
$$

From (55) and (57) it follows that $A(y)=\left(\lambda_{m}-t\right) y$, so $\left\|y^{\prime}\right\|_{2}^{2}=\left(\lambda_{m}-t\right)\|y\|_{2}^{2}$, hence $t\|y\|_{2}^{2}=0$; bearing in mind that $\|y\|=1$ we deduce that $t=0$.

Reasoning as in the proof of Proposition 3.4 (see (29) and (30)), we show that $\frac{\left\|\widehat{u}_{n}\right\|}{\left\|u_{n}\right\|^{\alpha}} \rightarrow 0$ and $\frac{\left\|\bar{u}_{n}\right\|}{\left\|u_{n}\right\|^{\alpha}} \rightarrow 0$ in $W$, hence $\frac{\left\|\widehat{u}_{n}+\bar{u}_{n}\right\|}{\left\|u_{n}\right\|^{\alpha}} \rightarrow 0$ in $W$ as $n \rightarrow \infty$. Then, if $R>0$ and $\eta \in(0,1)$ are as postulated by Proposition 3.3, we have

$u_{n} \in C_{\infty}(R, \eta, \alpha)=\left\{u \in W:\|u\| \geq R,\|\widehat{u}+\bar{u}\| \leq \eta\|u\|^{\alpha}\right\} \quad$ for all $n \geq n_{0}$, hence $\left\langle\varphi^{\prime}\left(u_{n}\right), u_{n}^{0}\right\rangle \geq \delta_{0}$ for all $n \geq n_{0}$. As $t_{n} \rightarrow 0^{+}$, we obtain

$$
\left\langle h_{t_{n}}^{\prime}\left(u_{n}\right), u_{n}^{0}\right\rangle \geq \frac{\delta_{0}}{2}>0 \quad \text { for all } n \geq n_{1} \geq n_{0},
$$

which contradicts (50). So, the Claim is true.

Note that $h_{0}=\varphi$ which satisfies the C-condition (see Proposition 3.4). In a similar way, exploiting the orthogonality of the component spaces, we check that $h_{1}$ too satisfies the C-condition. So, we can apply Proposition 2.1 and have

$$
C_{k}(\varphi, \infty)=C_{k}\left(h_{1}, \infty\right) \text { for all } k \geq 0 .
$$

From a slight modification of the proof of Proposition 3.10 of Bartsch and Li [5], we have

$$
C_{k}\left(h_{1}, \infty\right)=\delta_{k, d_{m}} \mathrm{Z} \text { for all } k \geq 0,
$$

hence

$$
C_{k}(\varphi, \infty)=\delta_{k, d_{m}} \mathrm{Z} \quad \text { for all } k \geq 0
$$

\section{Multiple nontrivial solutions}

In this section we prove the full multiplicity theorem for problem (1). First we show the existence of two constant sign solutions which are local minimizers of the energy functional $\varphi$. 
Proposition 4.1. If hypotheses $H$ hold, then problem (1) has two nontrivial constant sign solutions

$$
u_{0} \in \operatorname{int} C_{+}, v_{0} \in-\operatorname{int} C_{+}, \xi_{-}<v_{0}(t)<0<u_{0}(t)<\xi_{+} \quad \text { for all } t \in T
$$

and both are local minimizers of the energy functional $\varphi$.

Proof. Let $\theta \in(0,1)$ and consider the following truncation-perturbation of $f(t, x)$ :

$$
f_{+}^{\theta}(t, x)= \begin{cases}0 & \text { if } x \leq 0 \\ f(t, x)+\theta x & \text { if } 0<x<\xi_{+} \\ f\left(t, \xi_{+}\right)+\theta \xi_{+} & \text {if } \xi_{+} \leq x .\end{cases}
$$

We set $F_{+}^{\theta}(t, x)=\int_{0}^{x} f_{+}^{\theta}(t, s) d s$ and consider the functional $\varphi_{+}^{\theta}: W \rightarrow \mathbb{R}$ defined by

$$
\varphi_{+}^{\theta}(u)=\frac{1}{2}\left\|u^{\prime}\right\|_{2}^{2}+\frac{\theta}{2}\|u\|_{2}^{2}-\int_{0}^{b} F_{+}^{\theta}(t, u) d t \quad \text { for all } u \in W .
$$

We see that $\varphi_{+}^{\theta} \in C^{1,1}(W)$ and exploiting the compact embedding of $W$ into $C(T)$ we can check that $\varphi_{+}^{\theta}$ is sequentially weakly semicontinuous. Also, because of (59), clearly $\varphi_{+}^{\theta}$ is coercive. Therefore, invoking the Weierstrass theorem, we can find $u_{0} \in W$ such that

$$
\varphi_{+}^{\theta}\left(u_{0}\right)=\inf _{W} \varphi_{+}^{\theta}=m_{+}^{\theta} .
$$

First we show that $u_{0} \neq 0$. Indeed, hypotheses $H(\mathrm{v})$ implies that given $\varepsilon>0$, we can find $\delta=\delta(\varepsilon) \in\left(0, \xi_{+}\right)$such that

$$
f(t, x) \geq\left(\lambda_{i}-\varepsilon\right) x \text { for almost all } t \in T \text {, all } x \in[0, \delta],
$$

SO

$$
F(t, x) \geq \frac{1}{2}\left(\lambda_{i}-\varepsilon\right) x^{2} \text { for almost all } t \in T, \text { all } x \in[0, \delta] .
$$

Hence for $\xi \in[0, \delta] \subset\left[0, \xi_{+}\left[\right.\right.$and $\varepsilon \in\left(0, \lambda_{i}\right)$ (recall that $i \geq 1$ and so $\left.\lambda_{i}>0\right)$, we have

$$
\begin{aligned}
\varphi_{+}^{\theta}(\xi)=\frac{\theta \xi^{2}}{2} b-\int_{0}^{b} F_{+}^{\theta}(t, \xi) d t & =-\int_{0}^{b} F(t, \xi) d t \\
& \leq-\frac{1}{2}\left(\lambda_{i}-\varepsilon\right) x^{2} b<0,
\end{aligned}
$$

and from this we deduce

$$
\varphi_{+}^{\theta}\left(u_{0}\right)=\inf _{W} \varphi_{+}^{\theta}=m_{+}^{\theta}<0=\varphi_{+}^{\theta}(0),
$$

SO

$$
u_{0} \neq 0
$$

From (60), we have

$$
\left(\varphi_{+}^{\theta}\right)^{\prime}\left(u_{0}\right)=A\left(u_{0}\right)+\theta u_{0}-N_{+}^{\theta}\left(u_{0}\right)=0
$$

where $N_{+}^{\theta}(u)(\cdot)=f_{+}^{\theta}(\cdot, u(\cdot))$ for all $u \in W$, namely

$$
-u_{0}^{\prime \prime}(t)+\theta u_{0}(t)=f_{+}^{\theta}\left(t, u_{0}(t)\right) \text { a.e. on } T, u_{0}(0)=u_{0}(b), u_{0}^{\prime}(0)=u_{0}^{\prime}(b) \text {. }
$$


From (64) it follows that $u_{0} \in \widehat{C}(T) \backslash\{0\}$. In (63) we act with $\left(u_{0}-\xi_{+}\right)^{+} \in W$ and obtain

$$
\begin{aligned}
\left\langle A\left(u_{0}\right),\left(u_{0}-\xi_{+}\right)^{+}\right\rangle+\theta \int_{0}^{b} u_{0}\left(u_{0}-\xi_{+}\right)^{+} d t & =\int_{0}^{b} f_{+}^{\theta}\left(t, u_{0}\right)\left(u_{0}-\xi_{+}\right)^{+} d t \\
& =\int_{0}^{b}\left(f\left(t, \xi_{+}\right)+\theta \xi_{+}\right)\left(u_{0}-\xi_{+}\right)^{+} d t
\end{aligned}
$$

owing to hypothesis $H(\mathrm{vi})$ we achieve

$$
\left\langle A\left(u_{0}\right)-A\left(\xi_{+}\right),\left(u_{0}-\xi_{+}\right)^{+}\right\rangle+\theta \int_{0}^{b} u_{0}\left(u_{0}-\xi_{+}\right)^{+} d t \leq 0,
$$

hence $\theta\left\|\left(u_{0}-\xi_{+}\right)^{+}\right\|^{2} \leq 0$, so

$$
\left(u_{0}-\xi_{+}\right)^{+}=0 \text {, i.e., } u_{0}(t) \leq \xi_{+} \text {for all } t \in T \text {. }
$$

Also, on (64) we act with $-u_{0}^{-} \in W$, use (60) and obtain $\left\|\left(u_{0}^{-}\right)^{\prime}\right\|_{2}^{2}+\theta \|\left(u_{0}^{-} \|_{2}^{2}=\right.$ 0 , so $\theta\left\|u_{0}^{-}\right\| \leq 0$, and finally

$$
u_{0}(t) \geq 0 \text { for all } t \in T, u_{0} \neq 0(\text { see }(62)) .
$$

From the last two equations we see that $u_{0}(t) \in\left[0, \xi_{+}\right]$for all $t \in T$ and in this case (60) yields

$$
-u_{0}^{\prime \prime}(t)=f\left(t, u_{0}(t)\right) \text { a.e. on } T, u_{0}(0)=u_{0}(b), u_{0}^{\prime}(0)=u_{0}^{\prime}(b),
$$

hence $u_{0} \in C_{+} \backslash\{0\}$ and solves problem (1).

Hypotheses $H$ (ii), (iii) imply that there is $\sigma_{0}>0$ such that for a.a. $t \in T, x \rightarrow$ $f(t, x)+\sigma_{0} x$ is nondecreasing on $\left[\xi_{-}, \xi_{+}\right]$. Hence

$$
-u_{0}^{\prime \prime}(t)+\sigma_{0}\left(u_{0}(t)\right)=f\left(t, u_{0}(t)\right)+\sigma_{0}\left(u_{0}(t)\right) \geq 0 \text { a.e. on } T,
$$

so $u_{0}^{\prime \prime}(t) \leq \sigma_{0}\left(u_{0}(t)\right)$ a.e. on $T$ and from this we infer (see [21])

$$
u_{0} \in \operatorname{int} C_{+} \cdot
$$

Similarly, using hypotheses $H(\mathrm{vi})$ and the fact that $u_{0} \leq \xi_{+}$we obtain

$$
\begin{aligned}
& -\left(\xi_{+}-u_{0}\right)^{\prime \prime}(t)+\sigma_{0}\left(\xi_{+}-u_{0}(t)\right)=f\left(t, \xi_{+}\right)+\sigma_{0}\left(\xi_{+}\right)-f\left(t, u_{0}(t)\right)-\sigma_{0}\left(u_{0}(t)\right) \\
& \quad \geq 0 \text { a.e. on } T, \\
& \text { so }\left(\xi_{+}-u_{0}\right)^{\prime \prime}(t) \leq \sigma_{0}\left(\xi_{+}-u_{0}(t)\right) \text { a.e. on } T \text { and from this we deduce } \\
& \qquad \xi_{+}-u_{0} \in \operatorname{int} C_{+} .
\end{aligned}
$$

Hence from (67) and (68) it follows that $0<u_{0}(t)<\xi_{+}$for all $t \in T$, so

$$
u_{0} \in \operatorname{int}_{\widehat{C}(T)}\left[0, \xi_{+}\right]=i n t_{\widehat{C}(T)}\left\{u \in \widehat{C}(T): 0 \leq u_{0}(t) \leq \xi_{+} \text {for all } t \in T\right\} .
$$

Note that $\varphi_{\mid\left[0, \xi_{+}\right]}=\varphi_{+\mid\left[0, \xi_{+}\right]}^{\theta}$. So, from (69) we infer that $u_{0}$ is a local $\widehat{C}(T)$ minimizer of $\varphi$. Then from Proposition 5 of Papageorgiou and Papalini [16], it follows that $u_{0}$ is also a local $W$-minimizer of $\varphi$.

Similarly, if we truncate $x \rightarrow f(t, x)+\theta x$ at $\left\{\xi_{-}, 0\right\}$, as above we obtain another constant sign solution $v_{0} \in \operatorname{int} C_{+}, \xi_{-}<v_{0}(t)<0$ for all $t \in T$, which is also a local minimizer of the energy functional $\varphi$. 
The next lemma, can be found in Aizicovici et al. [2], where it was proved for Neumann partial differential equations driven by the p-Laplacian differential operator.

\section{Lemma 4.1.}

(a) If $\underline{u}_{1}, \underline{u}_{2} \in W$ are lower solutions for problem (1), then $\underline{u}=$ $\max \left\{\underline{u}_{1}, \underline{u}_{2}\right\} \in W$ is a lower solution too.

(b) If $\bar{u}_{1}, \bar{u}_{2} \in W$ are upper solutions for problem (1), then $\bar{u}=$ $\max \left\{\bar{u}_{1}, \bar{u}_{2}\right\} \in W$ is an upper solution too.

This lemma leads to the existence of extremal solutions in the order intervals $\left[0, \xi_{+}\right]=\left\{u \in \widehat{C}(T): 0 \leq u(t) \leq \xi_{+}\right.$for all $\left.t \in T\right\}$ and $\left[\xi_{-}, 0\right]=\{u \in$ $\widehat{C}(T): \xi_{-} \leq u(t) \leq 0$ for all $\left.t \in T\right\}$.

Proposition 4.2. If hypotheses $H$ hold, then problem (1) has a biggest solution $u^{*} \in$ int $C_{+}$in $\left[0, \xi_{+}\right]$and a smallest solution $v^{*} \in-$ int $C_{+}$in $\left[\xi_{-}, 0\right]$.

Proof. Let

$$
\mathcal{S}_{+}=\left\{u \in W: u \neq 0, u \text { solves }(1), 0 \leq u(t) \leq \xi_{+} \text {for all } t \in T\right\} .
$$

From Proposition 4.1, we know that $\mathcal{S}_{+} \neq \emptyset$. Next we show that $\mathcal{S}_{+}$is upward directed, i.e., if $u_{1}, u_{2} \in \mathcal{S}_{+}$, then we can find $u \in \mathcal{S}_{+}$such that $u_{1} \leq u, u_{2} \leq u$. So, let $u_{1}, u_{2} \in \mathcal{S}_{+}$. Then by virtue of Lemma 4.1 (a), we have that $\underline{u}=$ $\max \left\{u_{1}, u_{2}\right\} \in W$ is a lower solution for problem (1). Because of hypothesis $H(\mathrm{vi}) \bar{u} \equiv \xi_{+}$is an upper solution for (1) and $\underline{u} \leq \bar{u}$. As before, let $\theta \in(0,1)$ and consider the truncation of $x \rightarrow f(t, x)+\theta x$ at $\left\{\underline{u}(t), \bar{u}=\xi_{+}\right\}$for a.a. $t \in T$. Then via the direct method, we obtain a solution $\widehat{u}_{0} \in[\underline{u}, \bar{u}]=\{u \in$ $W: \bar{u}(t) \leq u(t) \leq \xi_{+}$for all $\left.t \in T\right\}$ (see [2]). This proves that $\mathcal{S}_{+}$is outward directed.

Now, we show that $\mathcal{S}_{+}$has a maximal element for the pointwise ordering on $W$. To this end, let $C \subseteq \mathcal{S}_{+}$be a chain (i.e., a totally ordered subset of $\mathcal{S}_{+}$). From Dunford and Schwartz [8, p. 336], we know that we can find $\left\{u_{n}\right\}_{n \geq 1} \subseteq C$ such that $\sup C=\sup _{n \geq 1} u_{n}$. we have

$$
A\left(u_{n}\right)=N\left(u_{n}\right) \quad \text { for all } n \geq 1 .
$$

As, $0 \leq u_{n}(t) \leq \xi_{+}$for all $t \in T$, we can find $c_{4}>0$ such that $\left\|u_{n}^{\prime}\right\| \leq c_{4}$ for all $n \geq 1$, hence $\left\{u_{n}\right\}_{n \geq 1} \subseteq w$ is bounded. So, we may assume that

$$
u_{n} \rightarrow u \text { in } W, \quad \text { and } u_{n} \rightarrow u \text { in } C(T) \text { as } n \rightarrow \infty .
$$

Passing to the limit as $n \rightarrow \infty$ in (70) and using (71), we obtain $A(u)=N(u)$, so $u=\sup C \in \mathcal{S}_{+}$.

Invoking the Kuratowski-Zorn lemma, we infer that $\mathcal{S}_{+}$has a maximal element $u^{*} \in \mathcal{S}_{+}$. This is the biggest solution of problem (1) in the ordered interval $\left[0, \xi_{+}\right]$. Indeed, let $u \in \mathcal{S}_{+}$. Since $\mathcal{S}_{+}$is upward directed, we can find $\widehat{u} \in \mathcal{S}_{+}$ such that $u^{*} \leq \widehat{u}, u \leq \widehat{u}$. The maximality of $u^{*}$ implies that $\widehat{u}=u^{*}$. Hence $u \leq u^{*}$ and since $u \in \mathcal{S}_{+}$was arbitrary, we conclude the extremality of $u^{*}$. 
Similarly, if we consider the set

$$
\mathcal{S}_{-}=\left\{v \in W: v \neq 0, v \text { solves }(1), \xi_{-} \leq v(t) \leq 0 \quad \text { for all } t \in T\right\},
$$

then reasoning as above, we produce $v^{*} \in-i n t C_{+}$the smallest element of $\mathcal{S}$.

Now we are ready for the full multiplicity theorem for problem (1). Our proof combines variational methods with truncation techniques and Morse theory.

Theorem 4.1. If hypotheses $H$ hold, then problem (1) has at least six nontrivial smooth solutions $u_{0}, \widehat{u} \in$ int $C_{+}, \widehat{u}-u_{0} \in$ int $C_{+}, u_{0}(t)<\xi_{+}$for all $t \in T, v_{0}, \widehat{v} \in-\operatorname{int} C_{+}, v_{0}-\widehat{v} \in \operatorname{int} C_{+}, \xi_{-}<v_{0}(t)$ for all $t \in T$, and $y_{0}$, $\widehat{y} \in C^{1}(T)$.

Proof. Proposition 4.1 guarantees the existence of two smooth solutions of constant sign $u_{0} \in \operatorname{int} C_{+}, v_{0} \in-\operatorname{int} C_{+}$and $\xi_{-}<v_{0}(t)<0<u_{0}(t)<\xi_{+}$for all $t \in T$.

In fact, invoking Proposition 4.2, we may assume that $u_{0}$ (respectively $v_{0}$ ) is the biggest (respectively smallest) solution of (1) in the ordered interval $\left[0, \xi_{+}\right]$ (respectively $\left.\left[\xi_{-}, 0\right]\right)$. Let $\theta \in(0,1)$ and consider the following truncationperturbation of $f(t, x)$

$$
\widehat{f}_{+}^{\theta}(t, x)= \begin{cases}f\left(t, u_{0}(t)\right)+\theta u_{0}(t) & \text { if } x \leq u_{0}(t) \\ f(t, x)+\theta x & \text { if } u_{0}(t)<x .\end{cases}
$$

We set $\widehat{F}_{+}^{\theta}(t, x)=\int_{0}^{x} \widehat{f}_{+}^{\theta}(t, s) d s$ and define the functional $\widehat{\varphi}_{+}^{\theta}: W \rightarrow \mathbb{R}$ by

$$
\widehat{\varphi}_{+}^{\theta}(u)=\frac{1}{2}\left\|u^{\prime}\right\|_{2}^{2}+\frac{\theta}{2}\|u\|_{2}^{2}-\int_{0}^{b} \widehat{F}_{+}^{\theta}(t, u(t)) d t \quad \text { for all } u \in W .
$$

Note that $\hat{\varphi}_{+}^{\theta} \in C^{2-0}(W)$. Moreover, reasoning as in the proof of Proposition 3.4, we can check that $\widehat{\varphi}_{+}^{\theta}$ satisfies the C-condition.

Claim. $u_{0} \in \operatorname{int} C_{+}$is a local minimizer of $\widehat{\varphi}_{+}^{\theta}$. We truncate $\widehat{f}_{+}^{\theta}(t, \cdot)$ as follows

$$
\bar{f}_{+}^{\theta}(t, x)= \begin{cases}\widehat{f}_{+}^{\theta}(t, x) & \text { if } x \leq \xi_{+} \\ \widehat{f}_{+}\left(t, \xi_{+}\right) & \text {if } \xi_{+}<x .\end{cases}
$$

Set $\bar{F}_{+}^{\theta}(t, x)=\int_{0}^{x} \bar{f}_{+}^{\theta}(t, s) d s$ and consider the functional $\bar{\varphi}_{+}^{\theta}: W \rightarrow \mathbb{R}$ defined by

$$
\bar{\varphi}_{+}^{\theta}(u)=\frac{1}{2}\left\|u^{\prime}\right\|_{2}^{2}+\frac{\theta}{2}\|u\|_{2}^{2}-\int_{0}^{b} \bar{F}_{+}^{\theta}(t, u(t)) d t \quad \text { for all } u \in W .
$$

Clearly $\bar{\varphi}_{+}^{\theta} \in C^{2-0}(W)$ and from $(73)$ it is clear that $\bar{\varphi}_{+}^{\theta}$ is coercive. It is also sequentially weakly lower semicontinuous. So, by the Weierstrass theorem, we can find $\widehat{u}_{0} \in W$ such that $\bar{\varphi}_{+}^{\theta}\left(\widehat{u}_{0}\right)=\inf _{W} \bar{\varphi}_{+}^{\theta}=\bar{m}_{+}^{\theta}$, hence

$$
\left(\bar{\varphi}_{+}^{\theta}\right)^{\prime}\left(\widehat{u}_{0}\right)=A\left(\widehat{u}_{0}\right)+\theta \widehat{u}_{0}-\bar{N}_{+}^{\theta}\left(\widehat{u}_{0}\right)=0,
$$


where $\bar{N}_{+}^{\theta}(u)(\cdot)=\bar{f}_{+}^{\theta}(\cdot, u(\cdot))$ for all $u \in W$. On (74), first we act with $\left(u_{0}-\widehat{u}_{0}\right)^{+} \in W$. Bearing in mind (72), (73) and recalling that $u_{0}$ solves (1), we obtain

$$
\begin{aligned}
& \left\langle A\left(\widehat{u}_{0}\right),\left(u_{0}-\widehat{u}_{0}\right)^{+}\right\rangle+\theta \int_{0}^{b} \widehat{u}_{0}\left(u_{0}-\widehat{u}_{0}\right)^{+} d t=\int_{0}^{b} f\left(t, u_{0}\right)\left(u_{0}-\widehat{u}_{0}\right)^{+} d t \\
& \quad+\theta \int_{0}^{b} u_{0}\left(u_{0}-\widehat{u}_{0}\right)^{+} d t=\left\langle A\left(u_{0}\right),\left(u_{0}-\widehat{u}_{0}\right)^{+}\right\rangle+\theta \int_{0}^{b} u_{0}\left(u_{0}-\widehat{u}_{0}\right)^{+} d t,
\end{aligned}
$$

so

$$
\left\langle A\left(u_{0}\right)-A\left(\widehat{u}_{0}\right),\left(u_{0}-\widehat{u}_{0}\right)^{+}\right\rangle+\theta \int_{0}^{b}\left(u_{0}-\widehat{u}_{0}\right)\left(u_{0}-\widehat{u}_{0}\right)^{+} d t=0,
$$

hence $\theta\left\|\left(u_{0}-\widehat{u}_{0}\right)^{+}\right\|^{2} \leq 0$, so

$$
u_{0} \leq \widehat{u}_{0} .
$$

Next, on (74) we act with $\left(\widehat{u}_{0}-\xi_{+}\right)^{+} \in W$. Using (73), (72) and hypothesis $H$ (vi), we obtain

$$
\begin{aligned}
\left\langle A\left(\widehat{u}_{0}\right),\left(\widehat{u}_{0}-\xi_{+}\right)^{+}\right\rangle+\theta \int_{0}^{b} \widehat{u}_{0}\left(\widehat{u}_{0}-\xi_{+}\right)^{+} d t & =\int_{0}^{b}\left(f\left(t, \xi_{+}\right)+\theta \xi_{+}\right)\left(\widehat{u}_{0}-\xi_{+}\right)^{+} d t \\
& \leq \theta \int_{0}^{b} \xi_{+}\left(\widehat{u}_{0}-\xi_{+}\right)^{+} d t,
\end{aligned}
$$

so

$$
\left\langle A\left(\widehat{u}_{0}\right)-A\left(\xi_{+}\right),\left(\widehat{u}_{0}-\xi_{+}\right)^{+}\right\rangle+\theta \int_{0}^{b}\left(\widehat{u}_{0}-\xi_{+}\right)\left(\widehat{u}_{0}-\xi_{+}\right)^{+} d t \leq 0,
$$

hence $\theta\left\|\left(\widehat{u}_{0}-\xi_{+}\right)^{+}\right\|^{2} \leq 0$, so

$$
\widehat{u}_{0} \leq \xi_{+} .
$$

From (75) and (76) it follows that $u_{0}(t) \leq \widehat{u}_{0}(t) \leq \xi_{+}$for all $t \in T$ and so (74) becomes $A\left(\widehat{u}_{0}\right)=N\left(\widehat{u}_{0}\right)$, hence $-\widehat{u}_{0}^{\prime \prime}(t)=f\left(t, \widehat{u}_{0}(t)\right)$ a.e. on $T, \widehat{u}_{0}(0)=$ $\widehat{u}_{0}(b), \widehat{u}_{0}^{\prime}(0)=\widehat{u}_{0}^{\prime}(b)$, so $\widehat{u}_{0} \in \operatorname{int} C_{+}$solves (1) and $\widehat{u}_{0} \in\left[u_{0}, \xi_{+}\right]$. From this, due to the extremality of $u_{0}$ we deduce $\widehat{u}_{0}=u_{0}$. From (73) it is clear that $\left.\hat{\varphi}_{+}^{\theta}\right|_{\left[0, \xi_{+}\right]}=\left.\bar{\varphi}_{+}^{\theta}\right|_{\left[0, \xi_{+}\right]}$, while, from the proof of Proposition 4.1, we know that $u_{0} \in \operatorname{int}_{\widehat{C}(T)}\left[0, \xi_{+}\right]$; so, it follows that $\widehat{u}_{0}=u_{0}$ is a local $\widehat{C}(T)$-minimizer of $\widehat{\varphi}_{+}^{\theta}$, hence it is also a local $W$-minimizer of $\hat{\varphi}_{+}^{\theta}$ (see [16]). This proves the Claim. We may assume that $u_{0}$ is an isolated critical point of $\hat{\varphi}_{+}^{\theta}$. Indeed, suppose that we can find $\left\{u_{n}\right\}_{n \geq 1} \subseteq W$ such that $u_{n} \rightarrow u_{0}$ in $W$ as $n \rightarrow \infty$ and $\left(\widehat{\varphi}_{+}^{\theta}\right)^{\prime}\left(u_{n}\right)=0$ for all $n \geq 1$. Then, as above, we can check that $u_{0} \leq u_{n}$ for all $n \geq 1$ and $\left\{u_{n}\right\}_{n \geq 1} \subseteq$ int $C_{+}$. Then (72) implies that $u_{n}$ is a smooth solution of (1) for all $n \geq 1$ and so we have produced a whole sequence of distinct positive solutions of (1) and we are done. Then as in the proof of Proposition 6 of Motreanu et al. [14], we can find $\widehat{\rho}_{\theta}>0$ small such that

$$
\widehat{\varphi}_{+}^{\theta}\left(u_{0}\right)<\inf \left[\hat{\varphi}_{+}^{\theta}(u):\left\|u-u_{0}\right\|=\widehat{\rho}_{\theta}\right]=\widehat{m}_{\theta} .
$$


Let $\xi \in \mathbb{R}_{+}=[0,+\infty)$ with $\xi>\left\|u_{0}\right\|_{\infty}$. Then, owing to (72), we can find $c_{5}>0$ such that

$$
\widehat{\varphi}_{+}^{\theta}(\xi)=\frac{\theta}{2} \xi^{2} b-\int_{0}^{b} \widehat{F}_{+}^{\theta}(t, \xi) d t \leq c_{5}-\int_{0}^{b} F(t, \xi) d t .
$$

Since $\lambda_{m}>0$, given $\varepsilon \in\left(0, \lambda_{m}\right)$, we can find $\widehat{M}>0$ such that $F(t, x) \geq$ $\frac{1}{2}\left(\lambda_{m}-\varepsilon\right) x^{2}$ for a.a. $t \in T$, all $|x| \geq \widehat{M}$, so $F(t, x) \rightarrow+\infty$ uniformly for almost all $t \in T$ as $|x| \rightarrow \infty$. Hence from (78) we conclude that

$$
\widehat{\varphi}_{+}^{\theta}(\xi) \rightarrow-\infty \quad \text { as } \xi \rightarrow+\infty, \xi>0 \text {. }
$$

Because of (77), (79) and recalling that $\widehat{\varphi}_{+}^{\theta}$ satisfies the C-condition, we see that we can apply Theorem 2.1 and obtain $\widehat{u} \in W$ such that

$$
\widehat{\varphi}_{+}^{\theta}\left(u_{0}\right)=\widehat{m}_{\theta} \leq \widehat{\varphi}_{+}^{\theta}(\widehat{u}), \quad \text { and }\left(\widehat{\varphi}_{+}^{\theta}\right)^{\prime}(\widehat{u})=0 .
$$

From (80) we have that $\widehat{u} \neq u_{0}$ and

$$
A(\widehat{u})+\theta \widehat{u}=\widehat{N}_{+}^{\theta}(\widehat{u}) \text {, where } \widehat{N}_{+}^{\theta}(u)(\cdot)=\widehat{f}_{+}^{\theta}(\cdot, u(\cdot)) \text { for all } u \in W .
$$

On (81) we act with $\left(u_{0}-\widehat{u}\right)^{+} \in W$ and as before show that $u_{0} \leq \widehat{u}$ and $\widehat{u} \in \operatorname{int} C_{+}$. Hence $\widehat{u} \in \operatorname{int} C_{+}$is a smooth solution of (1) (see 72). Hypotheses $H$ (ii), (iii) imply the existence of a $\widehat{\sigma}>0$ such that for almost all $t \in$ $T, x \rightarrow f(t, x)+\widehat{\sigma} x$ is nondecreasing on $\left[-\|\widehat{u}\|_{\infty},\|\widehat{u}\|_{\infty}\right]$. Hence $-\left(\widehat{u}-u_{0}\right)^{\prime \prime}(t)+$ $\widehat{\sigma}\left(\widehat{u}-u_{0}\right)(t)=f(t, \widehat{u}(t))+\widehat{\sigma} \widehat{u}(t)-f\left(t, u_{0}(t)\right)-\widehat{\sigma} u_{0}(t) \geq 0$ a.e. on $T$, so $\left(\widehat{u}-u_{0}\right)^{\prime \prime}(t) \leq \widehat{\sigma}\left(\widehat{u}-u_{0}\right)(t)$ a.e. on $T$, hence $\widehat{u}-u_{0} \in \operatorname{int} C_{+}$(see [21]).

Similarly, if we use the other solution $v_{0} \in-i n t C_{+}$and the following truncation-perturbation of $f(t, x)$

$$
\widehat{f}_{-}^{\theta}(t, x)=\left\{\begin{array}{lll}
f(t, x)+\theta x & \text { if } & x<v_{0}(t) \\
f\left(t, v_{0}(t)\right)+\theta v_{0}(t) & \text { if } & v_{0}(t) \leq x,
\end{array}\right.
$$

then working as above, using this time (82), we obtain a second negative smooth solution $\widehat{v} \in-$ int $C_{+}$such that $v_{0}-\widehat{v} \in$ int $C_{+}$.

From Proposition 4.1 we know that $u_{0}$ and $v_{0}$ are both local minimizers of $\varphi$. Hence (see [13, p. 175])

$$
C_{k}\left(\varphi, u_{0}\right)=C_{k}\left(\varphi, v_{0}\right)=\delta_{k, 0} \mathrm{Z} \quad \text { for all } k \geq 0 .
$$

From the previous arguments, we know that $\widehat{u} \in$ int $C_{+}$is a critical point of mountain pass type for the functional $\widehat{\varphi}_{+}^{\theta}$, hence $C_{k}\left(\widehat{\varphi}_{+}^{\theta}, \widehat{u}\right) \neq 0$ (see [6]). Moreover, from (72) we have for every $u \in W, u \geq u_{0}$

$$
\begin{aligned}
\widehat{\varphi}_{+}^{\theta}(u) & =\frac{1}{2}\left\|u^{\prime}\right\|_{2}^{2}-\frac{\theta}{2}\left\|u_{0}\right\|_{2}^{2}-\int_{0}^{b} F(t, u) d t+\int_{0}^{b}\left(F\left(t, u_{0}\right)-f\left(t, u_{0}\right)\right) d t \\
& =\varphi(u)+c_{6}, \text { where } c_{6}=-\frac{\theta}{2}\left\|u_{0}\right\|_{2}^{2}+\int_{0}^{b}\left(F\left(t, u_{0}\right)-f\left(t, u_{0}\right)\right) d t .
\end{aligned}
$$

Hence, since $\widehat{u} \geq u_{0}$ we have

$$
C_{k}\left(\widehat{\varphi}_{+}^{\theta}, \widehat{u}\right)=C_{k}\left(\varphi+c_{6}, \widehat{u}\right)=C_{k}(\varphi, \widehat{u}) \quad \text { for all } k \geq 0,
$$

so,

$$
C_{k}(\varphi, \widehat{u}) \neq 0
$$


Recall that $\varphi \in C^{2}(W)$ and

$$
\left\langle\varphi^{\prime \prime}(\widehat{u}) y, v\right\rangle=\int_{0}^{b} y^{\prime} v^{\prime} d t-\int_{0}^{b} f_{x}^{\prime}(t, \widehat{u}) y v d t \quad \text { for all } y, v \in W .
$$

So, we can apply Proposition 25 of Bartsch [4] (see also [13, p. 195]) and have that

$$
C_{k}(\varphi, \widehat{u})=\delta_{k, 1} \mathrm{Z} \quad \text { for all } k \geq 0 .
$$

Similarly, we show that

$$
C_{k}(\varphi, \widehat{v})=\delta_{k, 1} \mathrm{Z} \quad \text { for all } k \geq 0 .
$$

From Proposition 3.5, we have

$$
C_{k}(\varphi, 0)=\delta_{k, d_{i}} \mathrm{Z} \quad \text { for all } k \geq 0\left(d_{i}=2 i+1\right)
$$

and from Proposition 3.6, we have

$$
C_{k}(\varphi, \infty)=\delta_{k, d_{m}} \mathrm{Z} \text { for all } k \geq 0\left(d_{m}=2 m+1\right) .
$$

Then (88) implies that we can find a critical point $y_{0} \in C^{1}(T)$ (regularity theory) of $\varphi$ that solves problem (1) and such that

$$
C_{d_{m}}\left(\varphi, y_{0}\right) \neq 0 \text {. }
$$

Because $m \geq 1, d_{m} \geq 3$ and since $i \neq m$ we also have $d_{i} \neq d_{m}$. Hence (89), together with (83), (85)-(87) implies $y_{0} \notin\left\{0, u_{0}, v_{0}, \widehat{u}, \widehat{v}\right\}$.

Let $\widehat{\mu}$ be the Morse index of $y_{0}$ and $\widehat{\nu}$ the nullity of $y_{0}$. From (89) and the Gromoll-Meyer theorem (see Sect. 2), we have $d_{m} \in[\widehat{\mu}, \widehat{\mu}+\widehat{\nu}]$ and $\widehat{\nu} \leq 2$ (recall that $d_{m}=2 m+1$ and $\widehat{\mu}, \widehat{\nu} \in \mathbb{N}_{0}$ ). If $\widehat{\nu}=0$, then $y_{0}$ is nondegenerate and so $C_{k}\left(\varphi, y_{0}\right)=\delta_{k, d_{m}} \mathrm{Z}$ for all $k \geq 0$. Also, if $\widehat{\nu}=1$, then by virtue of the shifting theorem (see [6, p. 333]) and Corollary 8.4(v), p. 194, of Mawhin and Willem [13], again we have $C_{k}\left(\varphi, y_{0}\right)=\delta_{k, d_{m}} \mathrm{Z}$ for all $k \geq 0$. Finally, if $\widehat{\nu}=2$, then for $d_{m}=\widehat{\mu}$ or $d_{m}=\widehat{\mu}+2$, we have $C_{k}\left(\varphi, y_{0}\right)=\delta_{k, d_{m}} \mathrm{Z}$ for all $k \geq 0$ (see [13]). So, assuming that $\left\{0, u_{0}, v_{0}, \widehat{u}, \widehat{v}, y_{0}\right\}$ are the only critical points, from the Morse theory with $t=-1$, we obtain $(-1)^{d_{i}}=0$, a contradiction. Finally, for $d_{m}=\widehat{\mu}+1$, from the Morse inequality (with $q=d_{m}+d_{1}=2(m+i+1)$ ) , we obtain $(-1)^{d_{i}} \geq 0$, hence $-1 \geq 0$, a contradiction again. Therefore, there is a critical point $\widehat{y}$ of $\varphi$ such that $\widehat{y} \notin\left\{0, u_{0}, v_{0}, \widehat{u}, \widehat{v}, y_{0}\right\}$. Then $\widehat{y} \in C^{1}(T)$ and solves problem (1).

\section{References}

[1] Aizicovici, S., Papageorgiou, N.S., Staicu, V.: Periodic solutions for second order differential inclusions with the scalar p-Laplacian. J. Math. Anal. Appl. 322, 913-929 (2006)

[2] Aizicovici, S., Papageorgiou, N.S., Staicu, V.: Existence of multiple solutions with precise sign informations for superlinear Neumann problems. Annali di Mat. Pura Ed Applicata 188, 679-719 (2009) 
[3] Bartolo, P., Benci, V., Fortunato, D.: Abstract critical point theorems and applications to some non linear problems with "strong" resonance et infinity. Nonlinear Anal. Theory Methods Appl. 7, 981-1012 (1983)

[4] Bartsch, T.: Critical point theory on partially ordered Hilbert spaces. J. Funct. Anal. 186, 117-152 (2001)

[5] Bartsch, T., Li, S.: Critical point theory on for asymptotically quadratic functionals and applications to problems with resonance. Nonlinear Anal. Theory Methods Appl. 28, 419-441 (1997)

[6] Chang, K.C.: Methods in Nonlinear Analysis. Springer, Berlin (2005)

[7] Dancer, E.N., Gupta, C.: A Liapunov-type result with applications to a Dirichlet-type two-point boundary value problem at resonance. Nonlinear Anal. 22, 305-318 (1994)

[8] Dunford, N., Schwarz, J.: Linear Operators I. Wiley, New York (1958)

[9] Gromoll, D., Meyer, W.: On differentiable functions with isolated critical points. Topology 8, 361-369 (1969)

[10] Gupta, C.: Two-point boundary value problem of Dirichlet-type with resonance at infinitely many eigenvalues. J. Math. Anal. Appl. 146, 501-511 (1990)

[11] Iannacci, R., Nkashama, M.N.: Nonlinear two-point boundary value problems at resonance without Landesmann-Lazer conditions. Proc. AMS 106, 943$952(1989)$

[12] Liang, Z., Su, J.: Multiple solutions for semilinear elliptic boundary value problems with double resonance. J. Math. Anal. Appl. 354, 147-158 (2009)

[13] Mawhin, J., Willem, M.: Critical Point Theory and Hamiltonian Systems. Springer, New York (1989)

[14] Motreanu, D., Motreanu, V., Papageorgiou, N.S.: A degree theoretic approach for multiple solutions of constant sign for nonlinear elliptic equations. Manuscr. Math. 124, 507-531 (2007)

[15] Papageorgiou, N.S., Kyritsi, S.: Handbook of Applied Analysis. Springer, New York (2009)

[16] Papageorgiou, N.S., Papalini, F.: Pairs of positive solutions for the periodic scalar p-Laplacian. J. Fixed Point Theory Appl. 5, 157-184 (2009)

[17] Perera, K., Schechter, M.: Solutions of nonlinear equations having asymptotic limits at zero and infinity. Calc. Var. PDES 12, 359-369 (2001)

[18] Sanchez, L.: Resonance problems with nonlinearity interfering with eigenvalues of higher order. Appl. Anal. 25, 275-286 (1987)

[19] Su, J., Li, H.: Multiplicity results for the two-point boundary value problems at resonance. Acta Math. Sci. B 26, 152-162 (2006) 
[20] Su, J., Zhao, L.: Multiple periodic solutions of ordinary differential equations with double resonance. Nonlinear Anal. 70, 1520-1527 (2009)

[21] Vazquez, J.: A strong maximum principle for some quasilinear elliptic equations. Appl. Math. Optim. 12, 191-202 (1984)

[22] Zou, W.: Multiple solutions results for two-point boundary value problem with resonance. Discrete Cont. Dyn. Syst. 4, 485-496 (1998)

Giuseppina Barletta

Dipartimento Patrimonio Architettonico e Urbanistico Facoltà di Architettura

Università di Reggio Calabria

Salita Melissari

89124 Reggio Calabria

Italy

e-mail: giuseppina.barletta@unirc.it

Nikolaos S. Papageorgiou

Department of Mathematics

National Technical University

Zagrafou Campus

15780 Athens

Greece

e-mail: npapg@math.ntua.gr

Received: 16 February 2011.

Accepted: 9 July 2011. 